University of Redlands

\title{
Orthorectifying Historical Imagery Using the Rational Function Model
}

\footnotetext{
A Major Individual Project submitted in partial satisfaction of the requirements for the degree of Master of Science in Geographic Information Systems

by

Wilmarie Cruz Marrero
}

Ruijin Ma, Ph.D., Committee Chair

Mark Kumler, Ph.D.

August 2012 
Orthorectifying Historical Imagery Using the Rational Function Model

Copyright (C) 2012

by

Wilmarie Cruz Marrero 
The report of Wilmarie Cruz Marrero is approved.

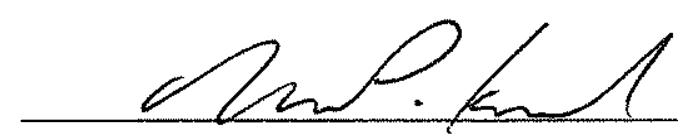

Mark Kumler, Ph.D.

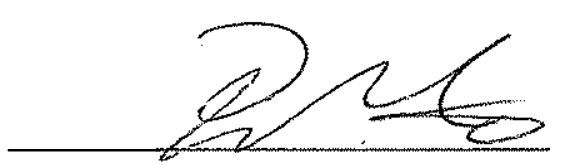

Ruijin Ma, Ph.D., Committee Chair

August 2012 


\section{Acknowledgements}

Apart from the efforts of myself, the success of any project depends largely on the encouragement and guidance of many others. I take this opportunity to express my gratitude to the people who have been instrumental in the successful completion of this project. I would like to show my greatest appreciation to the National GeospatialIntelligence Agency for selecting me to participate in this program.

I would also like to express my gratitude to Professor Ruijin Ma, committee chair and advisor for this project. He has put many hours of his time working to help me comprehend the project and assisting me to find solutions to the problems I ran into while doing my analysis. I would like to thank him for his patience and guidance throughout the months we were working together. Without his constant help I wouldn't have been able to finish my project.

I would also like to thank Professor Doug Flewelling for his continued support throughout the year in the University of Redlands MS GIS Program. Thanks for always being open to discuss frustrations and provide guidance for any problems we had.

To Debbie Riley, I appreciate everything you have done for me this past year. Debbie you made life easier here at the University of Redlands. Thank you for keeping us updated with all the academic logistics from workshops, to classes, and colloquiums.

To my partner in life Jamie Trout, you are the most important person in my life. I have never imagined that god was going to put in my path such an amazing person. I am sorry for not being with you this long year but I am really grateful that we are almost done with it and victorious. Thank you for constantly visiting me, encouraging me and helping me even with my homework, you are one of a kind. I am deeply sorry for the 
moments that I wasn't very patient. I miss our life together and I am ready to go back to it and be with you for the rest of my life.

Last but not least, I want to thank my new friends from Cohort 20. For all the support you have given me this past year. Mike Bostick thanks for being my personal Remote Sensing expert, I am immensely grateful. Ragna, Pon and Ari thanks for all the Python tutorials. Rocky thank you for being such a good friend you were always there for me when I needed to vent and go to Hangar. 


\begin{abstract}
Accuracy Assessment of the Rational Function Model Orthorectifying Technique

by

Wilmarie Cruz Marrero

Historical aerial imagery is a valuable resource for the research community to identify and measure changes on the earth's surface. Unfortunately, the majority of historical aerial imagery lacks spatial referencing; this lack of spatial reference makes it very challenging to analyze these imagery data within a GIS context. Additionally, georeferencing images in mountainous areas impose great difficulty due to the relief displacement in images. Ground control points were selected from unreferenced images, DEMs and DOQQs to derive rational function models. The accuracy of the orthorectification is assessed based on the derived rational function models and the resultant orthophotos.
\end{abstract}





\section{Table of Contents}

Chapter 1 - Introduction ........................................................................................................ 1

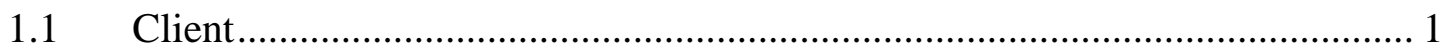

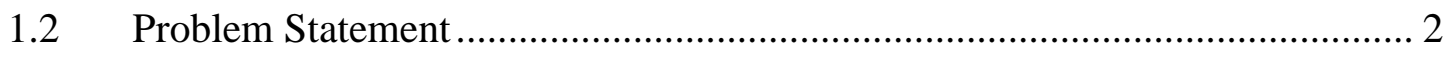

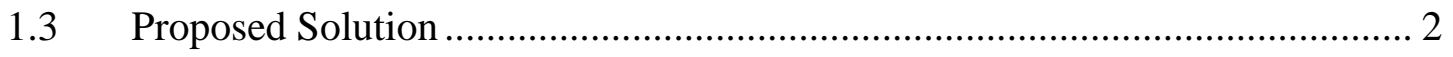

1.3.1 Goals and Objectives ........................................................................ 3

1.3 .2 Scope

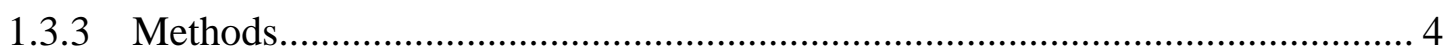

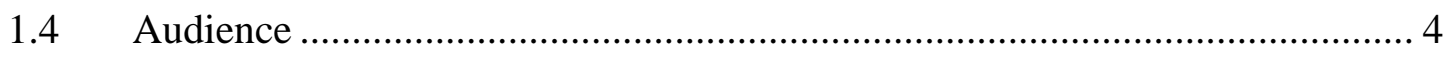

1.5 Overview of the Rest of this Report ........................................................ 4

Chapter 2 - Background and Literature Review .............................................................. 6

$2.1 \quad$ Importance of Historical Imagery ............................................................... 6

2.2 Geo-referencing vs. Orthorectification ..................................................... 7

2.3 Rational Function Model ....................................................................... 8

2.3.1 Rational Function Model Orders ……………….......................................... 10

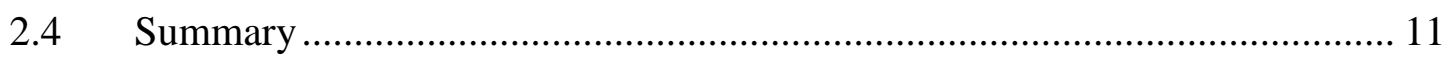

Chapter 3 - Systems Analysis and Design........................................................................... 12

3.1 Problem Statement ................................................................................ 12

3.2 Requirements Analysis ........................................................................ 12

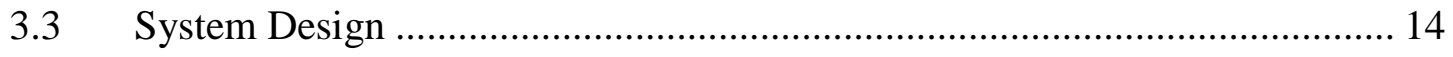

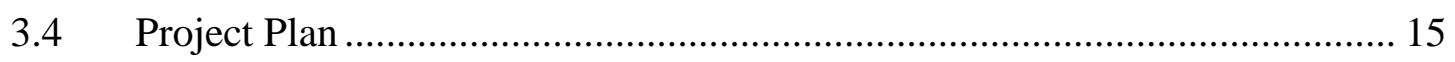

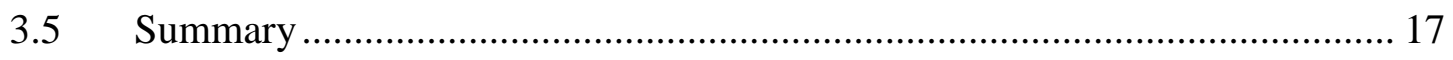


Chapter 4 - Database Design......................................................................................................... 19

4.1 Conceptual Data Model ........................................................................ 19

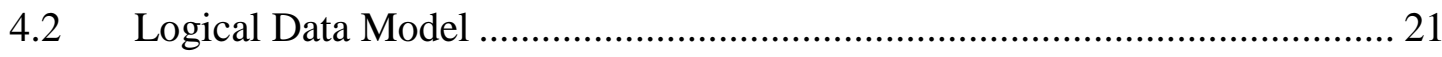

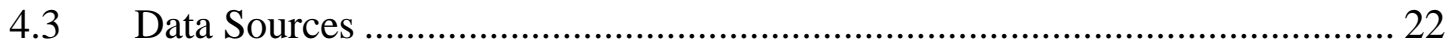

4.4 Data Collection Methods ……………………….................................. 23

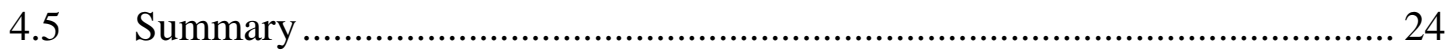

Chapter 5 - Implementation.............................................................................................. 25

$5.1 \quad$ Ground Control Points ................................................................................ 25

5.2 Rational Function Model Tools ………………………………….............. 29

5.3 Generating Orthophotos Using the Tool ...................................................... 31

5.4 Accuracy Assessment ............................................................................ 32

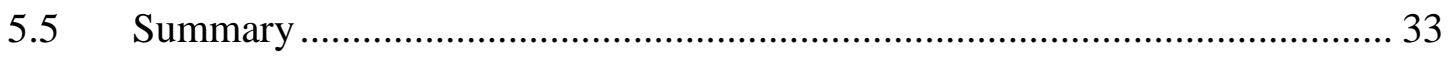

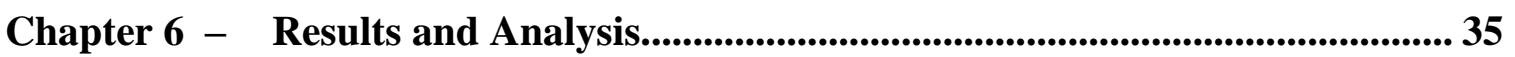

Chapter 7 - Conclusions and Future Work ....................................................................... 43

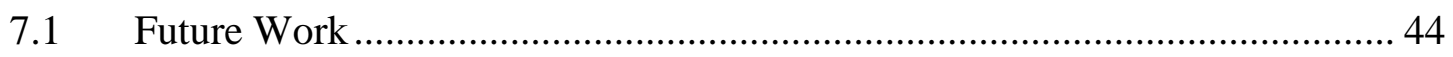

Works Cited $\quad$............................................................................

Appendix A. Python Script for RFM Bilinear Orthorectification Tool ...................... 47

Appendix B. Accuracy Spreadsheets.................................................................................... 51 


\section{Table of Figures}

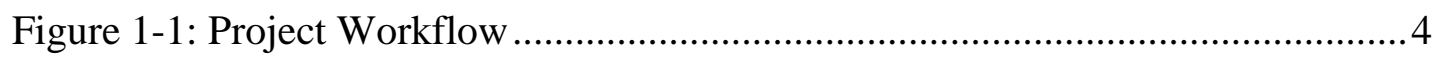

Figure 2-1: Method for developing the rational function model ..............................

Figure 3-1: System design of the project emphasizing the main components........... 15

Figure 4-1: Conceptual model UML diagram ..................................................... 19

Figure 4-2: More detailed look at the conceptual model of images ........................21

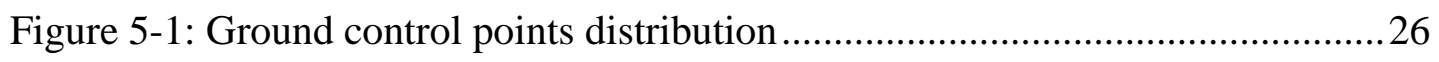

Figure 5-2: Ground control point text file ..................................................... 28

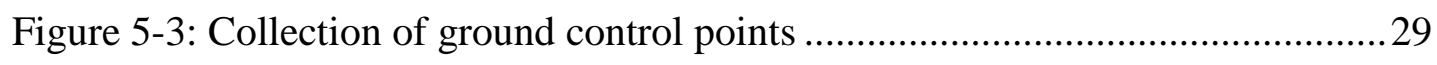

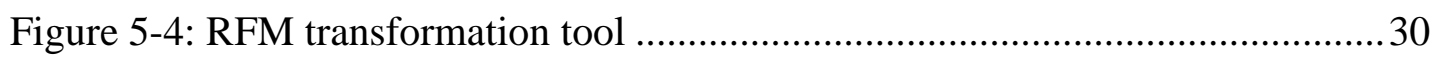

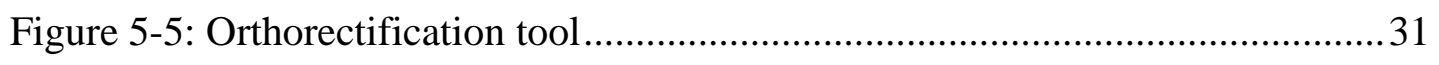

Figure 6-1: Riverside images with their respective RMSE values ........................ 41

Figure 6-3: Asymptote distortion created by RFM third order ...............................39

Figure 6-4: Digitized roads from the DOQQ and the RFM orthoimage................... 40

RMSE of the RFM orthophoto and the DOQQ for image 035-075 .......................51 



\section{List of Tables}

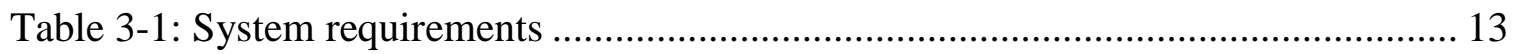

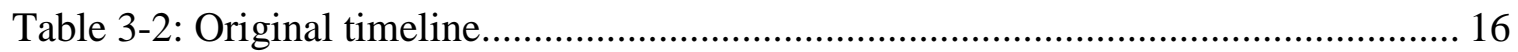

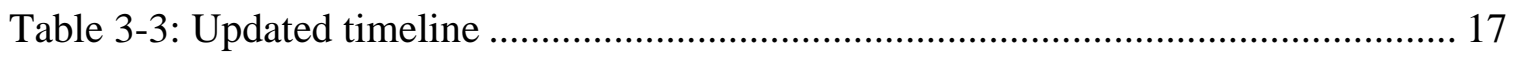

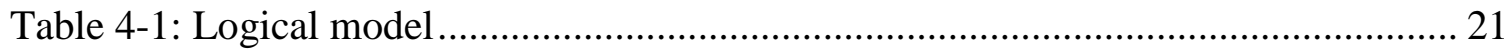

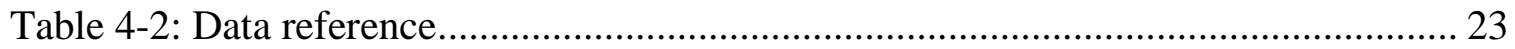

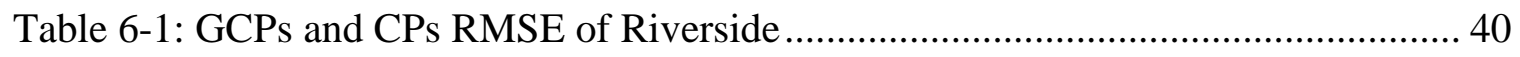

Table 6-2: RMSE of the RFM orthophoto and the DOQQ for images .......................... 42

Table 6-3: RMSE values for the GCPs used to calculate different RFM orders .............. 36

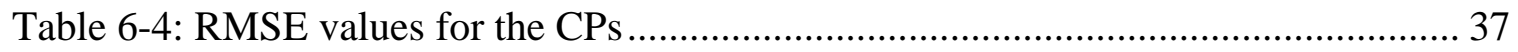

Table 6-5: RMSE values of GCPs collected over Texas .............................................. 35

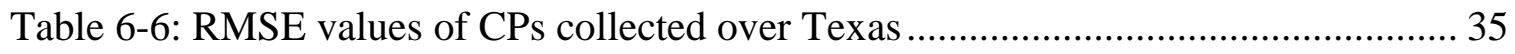





\section{List of Acronyms and Definitions}

CCB - Center for Conservation Biology

$\mathrm{CP}-$ Check points

DEM - Digital Elevation Model

DOQQ - Digital Orthophoto Quarter Quadrangle

GCP - Ground Control Points

GIS - Geographic Information Systems

GDAL - Geospatial Data Abstraction library

NAD- North American Datum

NOAA- National Oceanic and Atmospheric Administration

RFM - Rational Function Model

RMSE - Root Mean Squared Error

UML- Unified Modified Language

USGS - United States Geological Survey

UTM- Universal Transverse Mercator 


\section{Chapter 1 - Introduction}

Historical aerial photography has become a widely used data resource in the research community and is used extensively when comparing and measuring changes in the Earth's surface. However, most historical aerial photography lack spatial reference. This lack of spatial reference makes it very challenging to analyze these imagery data within a GIS context. The Center for Conservation Biology at the University of California, Riverside (CCB) in its ongoing research has a collection of historical imagery that can't be used to perform analysis because of their lack of spatial reference. Additionally, the majority of the images cover rough mountain terrain areas, making them difficult to georeference accurately. These photographs have to be orthorectified to achieve acceptable accuracy. The goal of this project is to conduct an accuracy assessment of the rational function model in orthorectifying historical aerial photographs and to verify how closely the rational function model can approximate the rigorous camera model.

\subsection{Client}

The clients for this project were Dr. Ruijin Ma, University of Redlands, and the Center for Conservation Biology at the University of California, Riverside. The CCB is comprised of researchers working to identify new study sites in conservation biology. Most of the researchers who use the imagery are not familiar with GIS, or have very limited computer skills. In addition to the researchers, the CCB also employs undergraduates to help with research who have only a few months of ArcGIS training. Ma developed different orders of rational function models (RFM) to orthorectify these historical photographs and he needed help conducting analyses of the accuracy of the 
RFMs in orthorectifying historical aerial images and research how accurately a rigorous camera model can approximate the RFM.

\subsection{Problem Statement}

The collection of unprocessed historic aerial imagery in the CCB lacks spatial reference. In order to be used for spatial analyses, the images need to be transformed to a spatial reference system. With no spatial reference for an image, it is difficult for researchers to identify which images covered their individual areas of study. Some of the aerial images also needed to be corrected from relief distortion for acceptable accuracy. The purpose of this research is to conduct an accuracy assessment of the rational function model, to investigate the accuracy of the different orders of RFMs.

\subsection{Proposed Solution}

The majority of the CCB's historical aerial imagery lacks spatial reference which made it very challenging to analyze these imagery data within a geographical information system (GIS) context. Additionally, raw images usually contain geometric distortions which introduce inaccuracies when used to conduct GIS analysis. Without the original camera information, the rational function model approach was selected because it can correct distortions caused by relief changes in rough mountain terrain. The problem was addressed with a two-part approach. The first step was to collect ground control points (GCP) and check points (CP) from different sets of aerial images in different types of terrain, mountainous regions and metropolitan areas. Then RFM coefficients were calculated from the collected GCPs using Python scripted tools. The second step was to orthorectify the unreferenced scanned aerial photographs to perform an accuracy assessment. 


\subsubsection{Goals and Objectives}

The goal of the project was to help the research community evaluate which rational function model was more accurate to orthorectify historical aerial imagery. The accuracy assessment test was conducted by measuring the RMSE values of ground control points and check points collected from each aerial photograph after they had been orthorectified and compared them to the same locations on the DOQQ.

\subsubsection{Scope}

The final deliverable of this project was an assessment that evaluates which RFM was most accurate for orthorectifying historical aerial imagery. The first component was to study different orders of the rational function model to orthorectify imagery. The results obtained from each RFM were compared with each other based on RMSE results. The second component was to document which of the RFMs was more accurate for historical imagery, and document that information for the client's benefit. The second component also included the installation and demonstration of the tools on the client computer system.

This project used black-and-white aerial photos acquired in 1938. The images were scanned at a resolution of 1200 dots per inch. The mission covers most of Riverside County, California which has mountainous areas. A second set of images was collected over suburban areas in Harris County, Texas. All imagery were provided by the client or were publicly available free data. All of the GCPs were measured from reference imagery, which means that the accuracy of the orthorectification relies on the accuracy of the reference imagery. 


\subsubsection{Methods}

The work for this part of the research project was conducted in ArcGIS 10. Ground control points (GCPs) and checkpoints (CPs) were collected using the identify tool from ArcGIS desktop toolbar matching the points on the elevation data, the DOQQ and the 1938 scanned images. Figure 1-1 shows the three data layers used in the collection of GCPs and CPs. After collecting the points a tool built using a Python script was used to calculate the RFM coefficients using the GCPs. After solving the model, the orthorectification of the imagery was performed based on its established rational function model. The resulting orthorectified images were used to test the accuracy of the RFMs based on their RMSE.
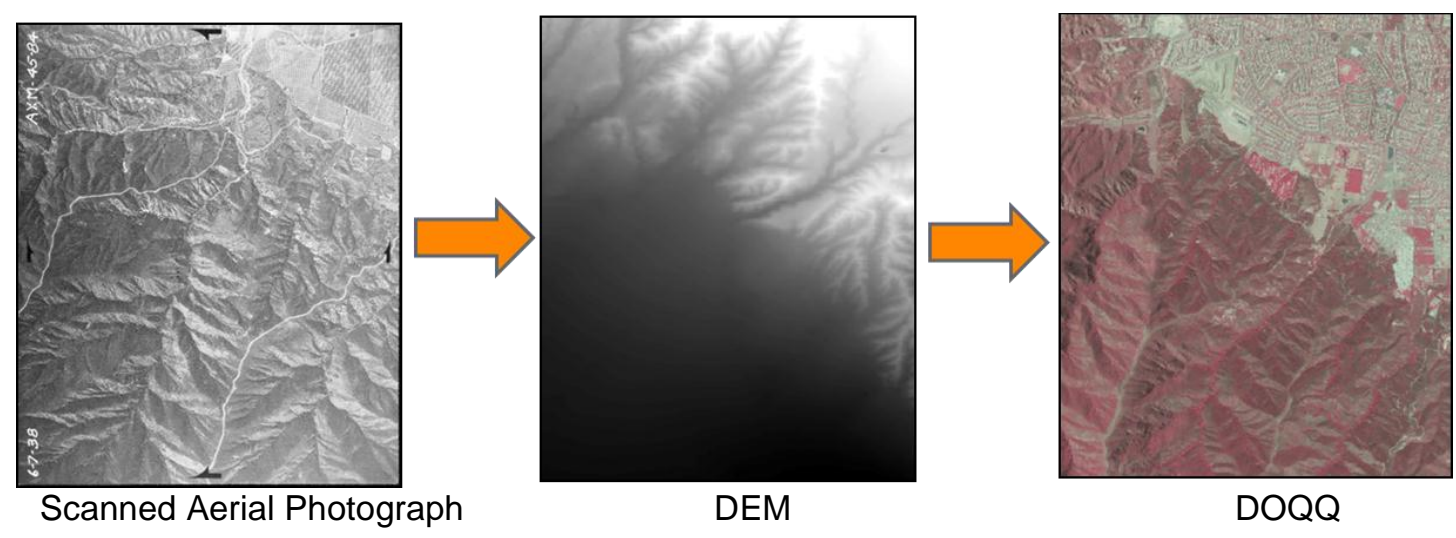

\section{Figure 1-1: Project Workflow}

\subsection{Audience}

This paper is intended to be read by the GIS specialist at the CCB. It discusses accurate methods of orthorectifying images using the rational function model in ArcGIS.

\subsection{Overview of the Rest of this Report}

Chapter Two is a literature review of previous research in the field discussing why aerial photography is important in conservation biology research. In addition to examining the 
use of aerial photography, Chapter two also explores the differences between georeferencing and orthorectifying imagery as well as an explanation of the RFM method. Chapter Three discusses the system analysis and design of the project including the overall project plan. Chapter Four outlines the database design, its conceptual and logical models. Chapter Five focuses on the steps that were taken to implement and complete the project. Chapter Six provides the results and an analysis of the overall project. Finally, Chapter Seven presents the project conclusions and possible future work. 


\section{Chapter 2 - Background and Literature Review}

Historical aerial imagery is a valuable resource for the research community to identify and measure changes on the Earth's surface. Unfortunately, the majority of historical aerial imagery lacks spatial reference which makes it very challenging to analyze imagery data within a GIS context (Buchwald, 2011). Geo-referencing imagery is one of the methods used to give an image its position in space, but geo-referencing an image covering a mountainous area imposes great difficulty due to relief displacement in images not modeled in geo-referencing. Orthorectification, however, is the process to correct geometric distortions caused by terrain variations. This chapter examines orthorectification techniques, as well as specifics of the rational function model, which is the method of choice for this project.

\subsection{Importance of Historical Imagery}

Historical aerial imagery has become a widely used resource in science, and is used extensively to compare and measure changes on the Earth's surface or different types of vegetation. Morgan, Gergel, and Coops (2010) describe the impact of aerial photography on environmental research as being "the longest-available, temporally continuous, and spatially complete record of landscape changes, dating from the early 1930s" (p.47). From vegetation alterations to changes in the Antarctic glaciers (Fox and Czifersky, 2008), there is an extensive variety of questions that can be answered using historical aerial imagery as a retrospective comparison tool.

Historical aerial imagery with high image resolution provides detailed information over the areas of study without the necessity of field work. Even though historical aerial imagery has many key qualities, most lack spatial reference. The lack of 
information makes it very challenging to work with this type of imagery for several reasons. First, it is very difficult to locate specific areas of study using historical aerial imagery without a coordinate system. Secondly, spatial reference information of aerial imagery is essential to perform any type of spatial analysis because it is the tool to align different data sources together.

\subsection{Geo-referencing vs. Orthorectification}

Geo-referencing imagery is one of the methods used to give an image its position in space. According to Sumerall and Lloyd (2001), geo-referencing transforms the X, Y coordinates of spatial data into real-world coordinates in a standardized reference system. Geo-referencing an image is an important step for various remote sensing and photogrammetric applications because it is indispensable for orthophoto generation (Tonolo and Poli, 2003). However, aerial and satellite images contain spatial distortions caused by terrain relief or a tilt of the sensor. The more topographically diverse the landscape, the more the distortion will be inherent in the image. Geo-referencing can correct for some distortions but not for relief displacement; orthorectification should be used for areas of high relief displacement because it models the imaging geometry which relates $3 \mathrm{D}$ ground space to $2 \mathrm{D}$ image space.

Orthorectification is the process that helps to correct for geometrical distortions in an image while adjusting for topographic relief. According to the ArcGIS Resource Center "orthorectification is the process of stretching the image to match the spatial accuracy of a map by considering location, elevation, and sensor information" (ESRI, 2011). It is considered more accurate than geo-referencing because it uses a Digital Elevation Model (DEM) to correct for relief displacement. Orthorectification is used in 
photogrammetry to reference images with high accuracy. It is usually complemented with a sensor model to represent the relationship between an object's 3-dimensional coordinates in real space, and its 2-dimensional coordinates in an image ( $\mathrm{Hu}, \mathrm{Tao} \&$ Croitoru, 2004). Generally there are two types of imaging geometry representations: a rigorous camera model and a rational function model. For this research project we use the rational function model which uses the ratio of two polynomials for transformation.

\subsection{Rational Function Model}

The rational function model is a mathematical generalized sensor model. It uses a ratio of two polynomials to relate object point coordinates $(\mathrm{X}, \mathrm{Y}$, and $\mathrm{Z}$ ) to image pixel coordinates. Ground control points can be used to solve such a model, which approximates the physical sensor model with high accuracy when selected carefully (Di et al., 2003). The first order equation for the RFM used for the project is shown below:

$$
\begin{aligned}
& x=\frac{a_{0}+a_{1} * \mathrm{X}+a_{2} * \mathrm{Y}+a_{3} * \mathrm{Z}}{1+c_{1} * \mathrm{X}+c_{2} * \mathrm{Y}+c_{3} * \mathrm{Z}} \\
& y=\frac{b_{0}+b_{1} * \mathrm{X}+b_{2} * \mathrm{Y}+b_{3} * \mathrm{Z}}{1+c_{1} * \mathrm{X}+c_{2} * \mathrm{Y}+c_{3} * \mathrm{Z}}
\end{aligned}
$$

Figure 2-1 shows two different ways to calculate rational function coefficients (RFC): the terrain-independent approach and the terrain-dependent approach. The terrain-independent approach develops rational function coefficients that can produce very high approximation accuracy. The control points are virtual points generated from physical sensor/camera models. 


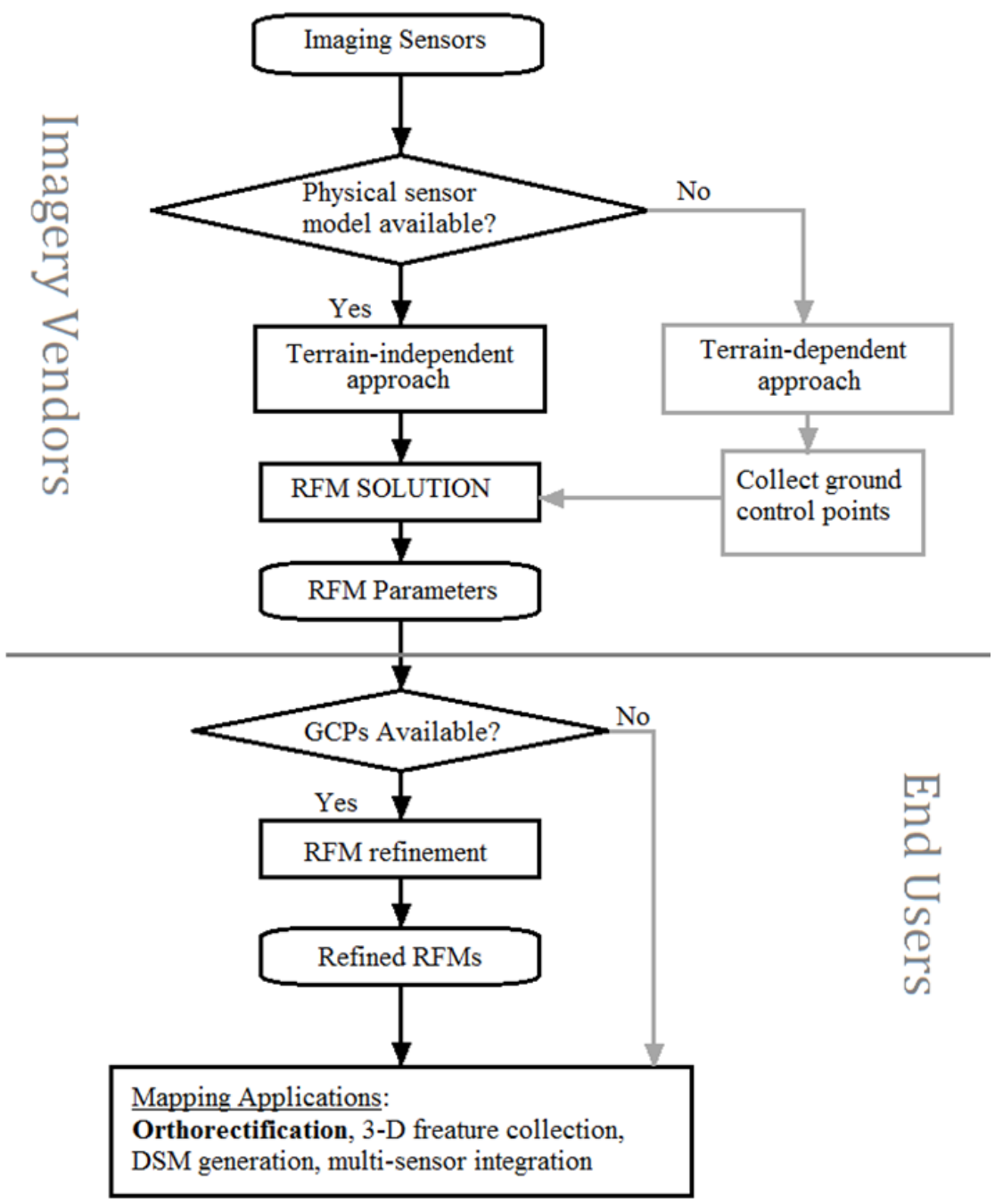

Figure 2-1: Method for developing the rational function model

The terrain-dependent approach is not as accurate as the terrain independent and is used when there is no rigorous sensor model available. It is very dependent on terrain relief, quality, and quantity of the GCPs. There must be a large number of GCPs, well distributed across entire area of the image (Tao and $\mathrm{Hu}, 2001)$. There are several techniques that help to properly select GCPs in the terrain dependent approach which could be utilized. According to Verbyla and Chang, (1997) ground control points should 
not be collected from areas that change, like shorelines, for example. Areas that have stationary features, like intersections or landmarks, are highly recommended.

According to Hu et al. (2004), if the sensor model information is unknown and the GCPs were collected properly, the terrain-dependent approach to the RFM can provide a better alternative to the 2-dimensional polynomial transformation that geo-referencing uses. The final approach to the RFM solution would be to properly design and implement a comprehensive test to evalute the accuracy of the RFM.

\subsubsection{Rational Function Model Orders}

There are generally three polynomial transformations used in RFM: 1st, 2nd, and 3rd order. Each uses a polynomial equation. The order of RFM refers to the order of the

polynomials it uses. In practice, the numerators or the denominators use the same order of polynomials. The 1st order polynomial uses a first degree linear equation, the 2 nd order uses a second degree equation that is parabolic and the 3rd order uses a 3rd degree equation known as a cubic equation (ESRI, 2011). Distortions that are caused by optical projections can be corrected using ratios of the first-order term, while corrections like Earth curvature and lens distortions can be approximated by the 2nd order polynomials terms. Other higher-order distortions could be corrected using 3rd terms (Tao and Hu, 2001).

Each order RFM requires a different minimum number of control points based on the amount of variables on them. In this project the denominators are the same for $\mathrm{X}$ and $\mathrm{Y}$ transformations. Thus, the 1 st order requires a minimum of six control points, the 2 nd order requires a minimum of fifteen control points and the 3rd order requires at least 
thirty control points. The collection of GCPs in the terrain dependent approach is very important because the solution is very sensitive to the GCP distribution.

\subsection{Summary}

Historical imagery is valuable and advantageous for identifying changes in land use, vegetation, and environmental conditions over different periods of time. However, most of the historical imagery lacks spatial reference. The scientific community needs help referencing images to conduct spatial analysis on them, and accurately research and analyze their particular areas of study. The RFM is the preferred method to orthorectify images due to its simplicity, standardized format, and high accuracy in rough mountain terrain when the camera information is not available. 


\section{Chapter 3 - Systems Analysis and Design}

This chapter discusses the overall design of the project and the requirements to solve the client's problem. It presents the requirements analysis, the system design, and the project plan used to solve the client's problem of assessing the accuracy of orthorectifying different types of images using three orders of the RFM.

\subsection{Problem Statement}

The CCB's collection of unprocessed historic aerial imagery lacked spatial reference. In order to be used for spatial analysis, the images needed to be transformed to a spatial reference system. Without geo-referencing an image, it is difficult for researchers to identify which images cover their individual areas of study. Some of the aerial images have severe relief distortion due to rough mountain terrain that also needs to be corrected. The purpose of the research was to conduct an accuracy assessment of the rational function model and investigate the accuracy of the different orders of the RFM in orthorectifying historical aerial photographs.

\subsection{Requirements Analysis}

The requirements analysis was based on the functions that were necessary to complete the project. Both the functional and non-functional requirements were discussed. The functional requirements were assessed according to the components necessary to complete the project. Non-functional requirements were focused on the technology for the user, as well as user restrictions and requirements that supported the main components. The functional and non-functional requirements are outlined in Table 3-1. 


\section{Table 3-1: System requirements}

\begin{tabular}{|l|l|l|}
\hline System Requirement & $\begin{array}{l}\text { Functional/Non- } \\
\text { Functional }\end{array}$ & Required/Optional \\
\hline $\begin{array}{l}\text { Data required to be } \\
\text { consistent with the CCB } \\
\text { data collection }\end{array}$ & Non-Functional & Required \\
\hline $\begin{array}{l}\text { Use ArcGIS desktop for the } \\
\text { processes }\end{array}$ & Non-Functional & Required \\
\hline $\begin{array}{l}\text { Calculate Rational Function } \\
\text { Model from control points }\end{array}$ & Functional & Required \\
\hline $\begin{array}{l}\text { Bi-linear image re-sampling } \\
\text { in orthorectification }\end{array}$ & Functional & Required \\
\hline Python 2.6 & Non-Functional & Required \\
\hline \hline GDAL image library & Non-Functional & Required \\
\hline
\end{tabular}

The main non-functional requirement was that everything should run in ArcGIS desktop 10 because the client had the same version of the software running on its computers and its interns were familiar with that version.

The main functional requirement was to conduct research on different approaches of orthorectification using the rational function model to find which RFM orders were more accurate. The research concepts were tested using historical aerial images. Since there were no pre-built tools to perform this process from ground control points (GCP), the tools needed to be scripted. The entire process was required by the client to be conducted in ArcGIS version 10. For that reason, the scripts made to perform the orthorectification had to be compatible with that version of the software. ArcGIS 10 
comes with Python and contains Python extensions, so the scripts had to be compatible with those as well. After Python and ArcGIS 10, the only other software-related functional requirement was a GDAL library. GDAL 18 had to be installed in the client's computer in order for the client to use the orthorectification tool.

\subsection{System Design}

The system was designed to conduct an accuracy assessment on different approaches to orthorectifying historical photographs using the rational function model to find which RFM order is more accurate. The main component of the design was a series of Python scripts that calculated the rational function model coefficients using the GCPs collected from the unreferenced image, DOQQ and DEM.

Since the camera geometry for the scanned images was not known, the coefficients for the rational function model had to be calculated from the manually collected GCPs. The design for this approach was to have the user generate control points from reference imagery DOQQs and DEMs and then run a script to calculate the coefficients. Once the coefficients were calculated, the orthorectification tool was used to transform the historical imagery to generate orthophotos. The tools were created from the script that runs in ArcMap and inserted on an ArcGIS toolbox to help the user perform the function easily with a standard ArcGIS graphic user interface.

Different types of tools were created. The first sets of tools were rational function model transformation tools with only two inputs: a text file with the ground control points, and the output file with the transformed coefficients for the specific order of the rational function model. These sets of tools were created to calculate the first, the second and the third order RFM. The second tool was an orthorectification tool that transforms 
unreferenced imagery into orthophotos using a DEM and the RFM coefficient file. Each tool has a help section in its respective interface with instructions explaining the use of the tool and the proper inputs and outputs to be used. Figure 3-1 shows the main components to successfully complete the RFM research project.

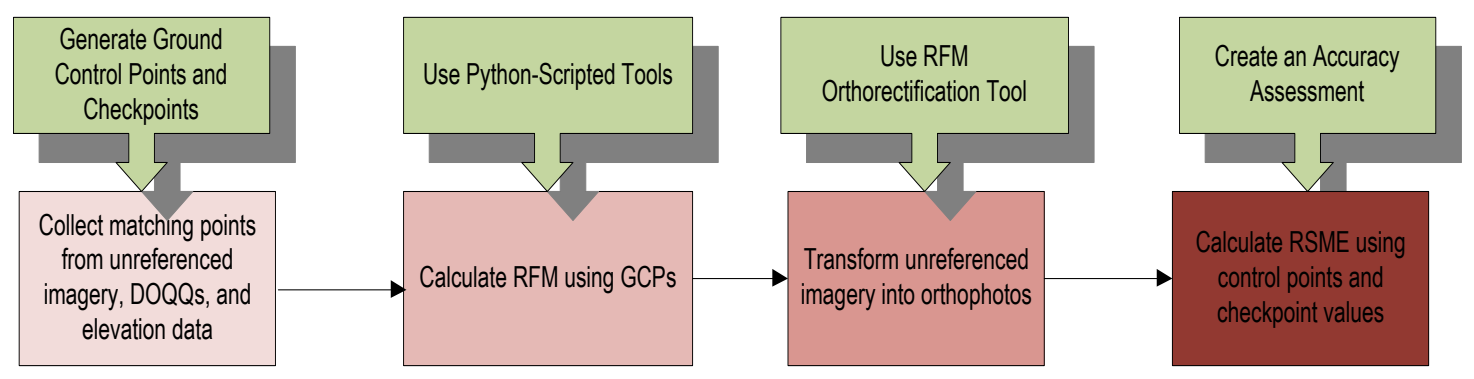

Figure 3-1: System design of the project emphasizing the main components

As part of the design tool, the ground control points file was designed as a text file to be used with the Python scripted tools. A key part in the process was using the correct format for the ground control points since the transformation tool read the ground control points in a very specific format.

\subsection{Project Plan}

The project changed from what was originally outlined in the project plan at the start of the program. The initial plan was to create a generic method using the RFM to orthorectify different types of imagery. The second component of the project was to research and investigate quadratic RFMs for more accurate referencing results. The third component was to explore other resampling methods to interpolate pixel values. There were two challenges anticipated. The first one was time constrained. The second one was the lack of proficiency on Python scripting. Table 3-2 shows the original project timeline. 
Table 3-2: Original timeline

\begin{tabular}{|c|c|c|c|c|c|c|c|c|c|c|c|c|}
\hline TASKS & Sep & Oct & Nov & Dec & Jan & Feb & Mar & Apr & May & Jun & Jul & Aug \\
\hline Planning & $\mathbf{x}$ & $\mathbf{x}$ & $\mathbf{x}$ & $\mathbf{x}$ & $\mathbf{x}$ & $\mathbf{x}$ & & & & & & \\
\hline Research & $\mathbf{x}$ & $\mathbf{x}$ & $\mathbf{x}$ & $\mathbf{x}$ & $\mathbf{x}$ & $\mathbf{x}$ & & & & & & \\
\hline Tool creation & & & & $\mathbf{x}$ & $\mathbf{x}$ & $x$ & $\mathbf{x}$ & $\mathbf{x}$ & & & & \\
\hline $\begin{array}{c}\text { Accurate } \\
\text { Assessment }\end{array}$ & & & & & & & & $\mathbf{x}$ & $\mathbf{x}$ & & & \\
\hline $\begin{array}{c}\text { Tool } \\
\text { Deployment }\end{array}$ & & & & & & & & & $\mathbf{x}$ & & & \\
\hline
\end{tabular}

The research project planned timeline changed throughout the duration of the year. Some tasks required more time due to unexpected delays, mistakes and failures that occurred while conducting the project.

The project was modified to perform an accuracy assessment of RFMs and to develop an orthorectification tool using the bi-linear image re-sampling method. The idea behind the project was to collect ground control points (GCPs) and checkpoints (CPs) in different types of images, to evaluate the accuracy of RFM in orthorectifying historical photographs. The GCPs were used to calculate different orders of the rational function model and then the unreferenced aerial images were orthorectified using the calculated RFMs. The orthorectification results and the root mean square error (RMSE) results of control points and checkpoints were calculated to conduct an accuracy assessment of the RFM, between different types of images. The accuracy assessment was conducted by comparing the RMSE, of different orders of RFMs, to assess which transformation method was more accurate. 
Table 3-3 shows the updated timeline where the black x's show the duration of each task. The red x's indicate tasks that exceeded the original time frame. The planning and research component were both too optimistic when originally planned. The time extension was necessary to ensure the project was done correctly. In addition, the tool creation step took more time because of the change in the project and a long period of time spent in learning Python. The project used a waterfall method. Components were delayed if an earlier component was stalled, like in the project completion and the tool deployment.

\section{Table 3-3: Updated timeline}

\begin{tabular}{|c|c|c|c|c|c|c|c|c|c|c|c|c|}
\hline TASK & Sep & Oct & Nov & Dec & Jan & Feb & Mar & Apr & May & Jun & Jul & Aug \\
\hline Planning & $\mathbf{x}$ & $x$ & $\mathbf{x}$ & $\mathbf{x}$ & $x$ & $\mathbf{x}$ & $x$ & $x$ & $x$ & $x$ & & \\
\hline Research & $\mathbf{x}$ & $\mathbf{x}$ & $\mathbf{x}$ & $\mathbf{x}$ & $\mathbf{x}$ & $\mathbf{x}$ & $x$ & $\mathbf{x}$ & $x$ & $\mathbf{x}$ & & \\
\hline Tool creation & & & & & & & $\mathbf{x}$ & $\mathbf{x}$ & $\mathbf{x}$ & $\mathbf{x}$ & & \\
\hline Orthorectifying & & & & & & & & $\mathbf{x}$ & $\mathbf{x}$ & $x$ & & \\
\hline $\begin{array}{c}\text { Accurate } \\
\text { Assessment }\end{array}$ & & & & & & & & & $\mathbf{x}$ & $x$ & $\mathbf{x}$ & \\
\hline $\begin{array}{c}\text { Tool } \\
\text { Deployment }\end{array}$ & & & & & & & & & & $\mathbf{x}$ & & \\
\hline $\begin{array}{c}\text { Project } \\
\text { Completion }\end{array}$ & & & & & & & & & & & & $\mathbf{x}$ \\
\hline Documentation & $\mathbf{x}$ & $x$ & $\mathbf{x}$ & $\mathbf{x}$ & $\mathbf{x}$ & $\mathbf{x}$ & $\mathbf{x}$ & $\mathbf{x}$ & $\mathbf{x}$ & $\mathbf{x}$ & $\mathbf{x}$ & $\mathbf{x}$ \\
\hline
\end{tabular}

\subsection{Summary}

This chapter discussed the system analysis and project functional and non-functional requirements. The functional requirements focused on the main components of the project, and the non-functional requirements focused on the environment for the developed tools to run successfully. The system design and project plan were also discussed in this chapter. The system design explained the major system components, the use of a series of Python scripts that calculate rational function model coefficients using 
the ground control points and two other scripts that orthorectified imagery to conduct an accuracy assessment of the RFM.

Although the project plan changed throughout the duration of the project, the overall concepts stayed the same. 


\section{Chapter 4 - Database Design}

Database design is one of the most important aspects of a GIS project. The conceptual and logical models designed for this project, as well as the data management and data sources, are discussed in this chapter.

\subsection{Conceptual Data Model}

The conceptual data model was based on the working components of the clients' needs and their relationship. This particular research project didn't require the creation of a database management system, instead a small geodatabase was created and used to store images to be orthorectified as well as the transformation and orthorectification Python scripted tools. Figure 4-1 shows the concept model of the database.

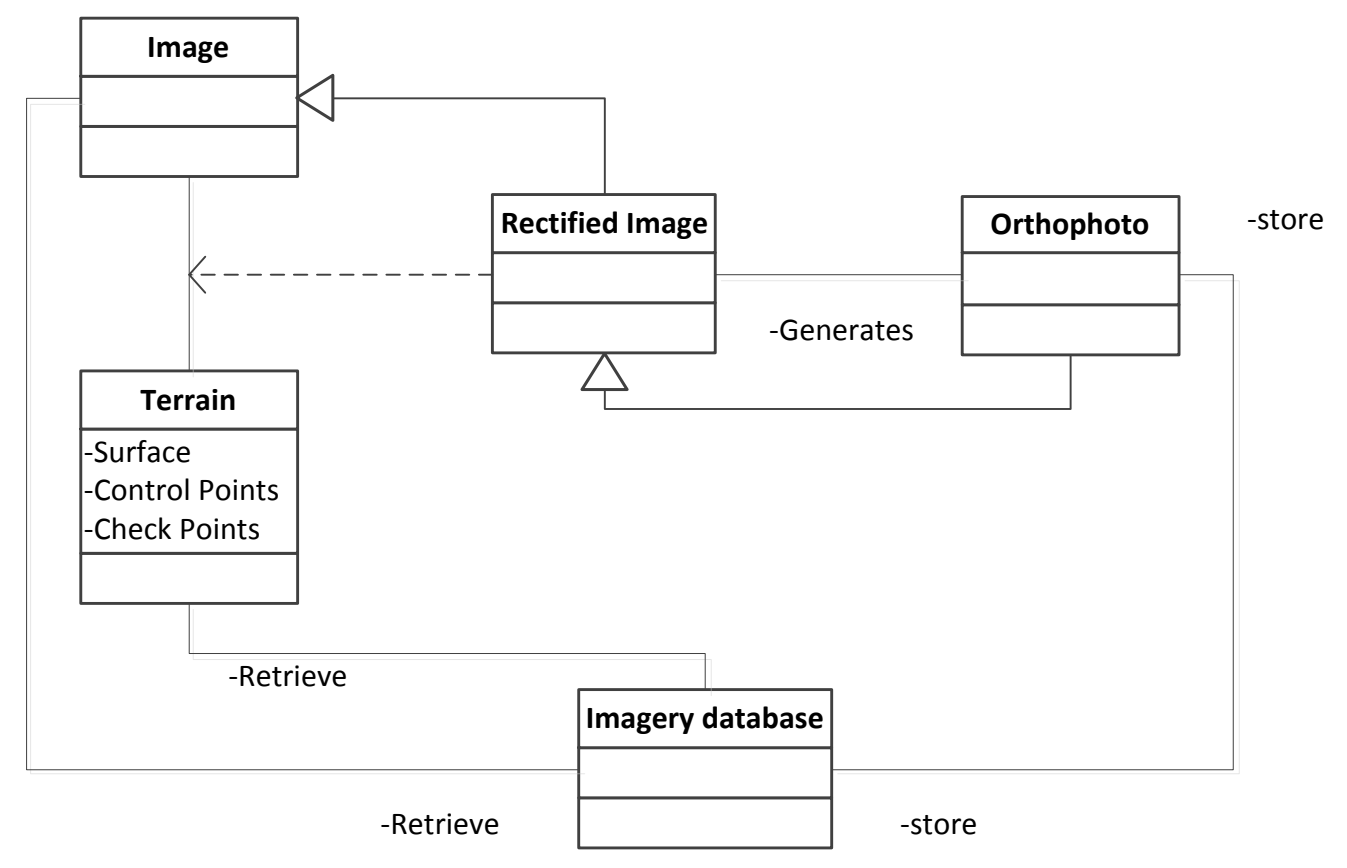

Figure 4-1: Conceptual model UML diagram 
The concept of this research project was to take a photo without any spatial reference information (coordinate system) and use an already referenced image to put the correct location of the unreferenced image on the ground.

Because of the nature of the project, the main goal was to create an accuracy assessment to identify effective orthorectification methods; the creation of a new database management system was not part of the requirements of the project. However the data to be transformed, the unreferenced historical imagery, DEM and DOQQ used in the project were stored in a database for easy retrieval and storage at the time of conducting analysis.

Figure 4-1, the conceptual model in Unified Modified Language (UML), shows the relationship between the various components of the database. Ground control points (GCPs) were used to conduct the transformation from ground coordinate system to image coordinate system. Once the transformation was established, the unreferenced images were orthorectified and stored in the database. The checkpoints were used to perform an independent accuracy assessment for the transformation.

Figure 4-2 displays the conceptual model of the imagery in more detail. The unreferenced imagery was rectified using the transformation established from the GCPs. It includes elevation data (Z-values), which was an important step in the creation of orthophotos. 


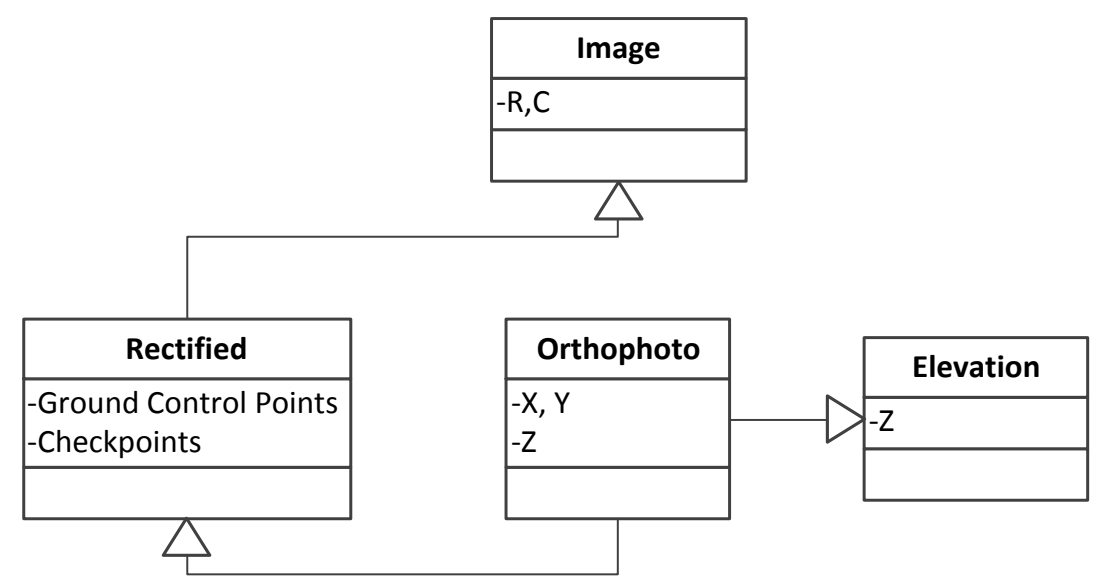

Figure 4-2: More detailed look at the conceptual model of images

\subsection{Logical Data Model}

The database of this project was simple, and the nature of the research project required only one geodatabase to be created. Tables 4-1 show the design of the database. It is composed of the three main components: external, internal, and outputs. The external components are inputs obtained from outside the system, some of which were provided by the clients. The internal component shows the tools used in the system to create the final product. The output component is the outcome of the tools and the final results. Each component was stored in the database.

\section{Table 4-1: Logical model}

\begin{tabular}{|ccc|}
\hline External & Internal & Output \\
\hline DEM & RFM Orthorectification tool & Orthorectified images \\
\hline DOQQ & Transformation tools & $\begin{array}{c}\text { Transformation } \\
\text { coefficients }\end{array}$ \\
\hline $\begin{array}{c}\text { Scanned aerial } \\
\text { photos }\end{array}$ & $\begin{array}{c}\text { Ground Control Points } \\
\text { Checkpoints }\end{array}$ & $\begin{array}{c}\text { Accuracy assessment } \\
\text { results }\end{array}$ \\
\hline GCP Text files & Accuracy assessment calculation & \\
& table & \\
\hline
\end{tabular}


The external components included the original images the client provided, unreferenced images, DOQQs and DEMs of areas over Harris County, Texas, and Riverside County, California. The internal components included: the transformation tools that transformed the GCPs to coefficients; the RFM orthorectification tool that orthorectify unreferenced imagery with DEM; the GCP files and the CP files; and the accuracy assessment calculation spreadsheet. All of these components were used to produce the final results.

The last component, the output, included the orthorectified images, the transformation coefficients used in the accuracy assessment calculations, and the accuracy assessment results.

\subsection{Data Sources}

The data for this project included the scanned aerial photographs, the DOQQs, the DEMs, and Python-scripted tools for the RFM calculations and orthorectification tool, ground control points and checkpoints collected over Harris County, Texas. The clients provided the aerial photographs and the control points collected over Harris County. The first set of images was black-and-white scanned aerial photographs of Riverside County, California, acquired in 1938 from AXM-1938 flight. The DEM are from the National Elevation Dataset, 2010 collection with geographic coordinates on the North American Datum of 1983. The DOQQS are high resolution orthophotos with spatial resolution of 1 meter. Both the 1993 black-and-white photos and the 1998 color infrared photos from USGS were used for reference with a spatial coordinate systems NAD 1983 UTM Zone 11 North. 
The second set of aerial photographs was collected over Harris County, Texas, in 2004.

The Texas data has rigorous camera models. Virtual control points were calculated using the rigorous camera model and photogrammetric aerial triangulation. Table 4-2 provides more information on the data used for the project.

\section{Table 4-2: Data reference}

\begin{tabular}{|c|c|c|c|c|c|c|}
\hline \multicolumn{2}{|c|}{ Data Set } & \multirow{2}{*}{$\begin{array}{l}\text { Acquisition } \\
2 / 27 / 2004 \\
\end{array}$} & \multirow{2}{*}{$\begin{array}{c}\text { Scale } \\
1 / 21600\end{array}$} & \multirow{2}{*}{$\begin{array}{c}\begin{array}{c}\text { Spatial } \\
\text { Resolution }\end{array} \\
0.3 \mathrm{~m}\end{array}$} & \multirow{2}{*}{$\begin{array}{c}\text { Number of } \\
\text { Control } \\
\text { Points }\end{array}$} & \multirow{2}{*}{$\begin{array}{c}\text { Number of } \\
\text { Check } \\
\text { Points }\end{array}$} \\
\hline Harris & 180223 & & & & & \\
\hline County, & 180224 & $2 / 27 / 2004$ & $1 / 21600$ & $0.3 \mathrm{~m}$ & 50 & 20 \\
\hline Texas & 552191 & $2 / 27 / 2004$ & $1 / 10800$ & $0.15 \mathrm{~m}$ & 50 & 20 \\
\hline & 552192 & $2 / 27 / 2004$ & $1 / 10800$ & $0.15 \mathrm{~m}$ & 50 & 20 \\
\hline \multirow{8}{*}{$\begin{array}{l}\text { Riverside } \\
\text { County, } \\
\text { California }\end{array}$} & 045_083 & 6/7/1938 & $1 / 20000$ & $0.42 \mathrm{~m}$ & 50 & 15 \\
\hline & $045-084$ & $6 / 7 / 1938$ & $1 / 20000$ & $0.42 \mathrm{~m}$ & 50 & 15 \\
\hline & 045_061 & $6 / 7 / 1938$ & $1 / 20000$ & $0.42 \mathrm{~m}$ & 20 & 15 \\
\hline & 045_063 & $6 / 7 / 1938$ & $1 / 20000$ & $0.42 \mathrm{~m}$ & 20 & 15 \\
\hline & 045_064 & 6/7/1938 & $1 / 20000$ & $0.42 \mathrm{~m}$ & 20 & 15 \\
\hline & 035_071 & $5 / 29 / 1938$ & $1 / 20000$ & $0.42 \mathrm{~m}$ & 20 & 15 \\
\hline & 035_073 & 5/29/1938 & $1 / 20000$ & $0.42 \mathrm{~m}$ & 20 & 15 \\
\hline & 035_075 & $5 / 29 / 1938$ & $1 / 20000$ & $0.42 \mathrm{~m}$ & 20 & 15 \\
\hline
\end{tabular}

\subsection{Data Collection Methods}

The data collection conducted in the project was the collection of GCPs and CPs in ArcGIS 10. The X-and Y-values were collected from the DOQQ, the Z-values were collected from the elevation data, the row and column numbers were measured from the unreferenced image. The values were organized in an X, Y, Z, COL, ROW format. They were put in a text file so the Python-scripted transformation tool could read and use them to calculate the desired RFM. Once the RFM coefficients were calculated, the unreferenced aerial images were orthorectified using the orthorectification tool.

Since the camera geometry for the 1938 historical aerial images from Riverside was not known, the GCPs and CPs for Riverside had to be manually collected using the 
identify tool from ArcGIS desktop 10. The points were collected using the 1938 images and the corresponding DOQQs and DEMs.

However, the Texas aerial imagery camera information is known because it has rigorous camera models. Virtual control points were calculated using the rigorous camera model and photogrammetric aerial triangulation.

\subsection{Summary}

Database design is one of the most important aspects in a GIS project. The nature of this project didn't require the creation of a new database management system because the main goal was to create an accurate assessment of the rational function model method. A geodatabase was created as an organized and efficient way to store and retrieve the imagery used to conduct the analyses. The project used data collected over rough mountain terrain of Riverside County, California with unknown camera geometry, and high resolution imagery collected over flat areas in Harris County, Texas with known camera values. 


\section{Chapter 5 - Implementation}

The purpose of the research was to conduct an accuracy assessment of the rational function model in order to investigate the accuracy of different orders of RFMs. When the sensor camera information is unknown, the RFM model coefficients need to be calculated using manually collected ground control points (GCP). The RFM equation used for this project was different from the basic RFM equation used by Buchwald (2011) in a previous research project. The basic equation for this project was normalized in order to have a unique solution. The normalization was achieved to have a constant number of 1 in the denominators. The linear RFM is shown below:

$$
\begin{aligned}
& x=\frac{a_{0}+a_{1} * \mathrm{X}+a_{2} * \mathrm{Y}+a_{3} * \mathrm{Z}}{1+c_{1} * \mathrm{X}+c_{2} * \mathrm{Y}+c_{3} * \mathrm{Z}} \\
& y=\frac{b_{0}+b_{1} * \mathrm{X}+b_{2} * \mathrm{Y}+b_{3} * \mathrm{Z}}{1+c_{1} * \mathrm{X}+c_{2} * \mathrm{Y}+c_{3} * \mathrm{Z}}
\end{aligned}
$$

The $\mathrm{X}, \mathrm{Y}$, and $\mathrm{Z}$ in the equation are ground coordinates collected from GCPs measured from unreferenced imagery, DOQQs, and elevation data. The a's, b's, and c's are the coefficients that need to be calculated from control points.

\subsection{Ground Control Points}

When the camera geometry is unknown, the RFM model coefficients need to be calculated from manually collected GCPs. The control points were taken as triplets from locations from the unreferenced photos, the orthophoto, and the elevation data. Figure 5-1 shows ground control points distribution. The $\mathrm{X}$ and $\mathrm{Y}$ values were collected from the 
USGS DOQQ; the Z values were collected from the USGS DEM; and the row and column from the unreferenced photo.

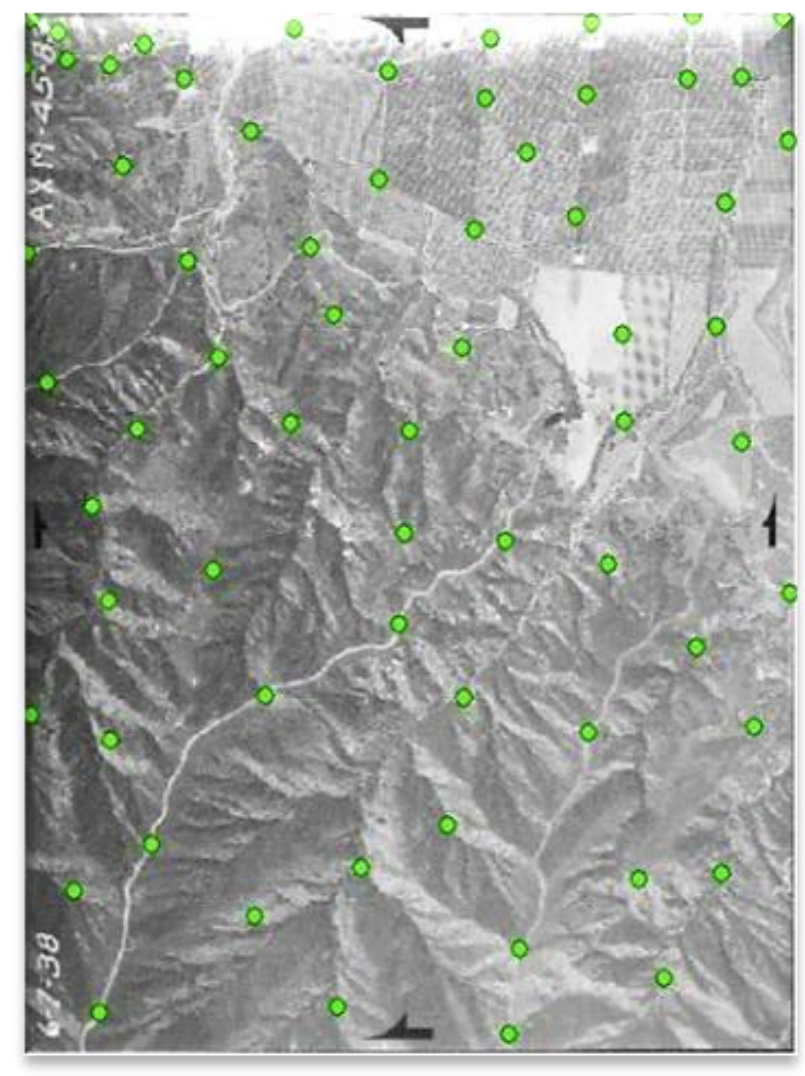

\section{Figure 5-1: Ground control points distribution}

The collection of ground control points was conducted in ArcGIS 10. GCPs were collected using the ArcGIS toolbar by matching the points on the DEM, DOQQ, and historical scanned-images. To collect the control points for the project the method used by Buchwald (2011) was followed. Two ArcMap documents were open at the same time: one with a 1938 image loaded and one with elevation data and a DOQQ. The DOQQ was the top layer because it was used as the reference layer, but the Identify tool was set to read the information from the elevation data. The same spot would be located and coordinates from both the 1938 image and the DOQQ image would be recorded, along 
with the elevation value at that location. Figure 5-2 shows an example of the collection of the GCPs using the three data layers.

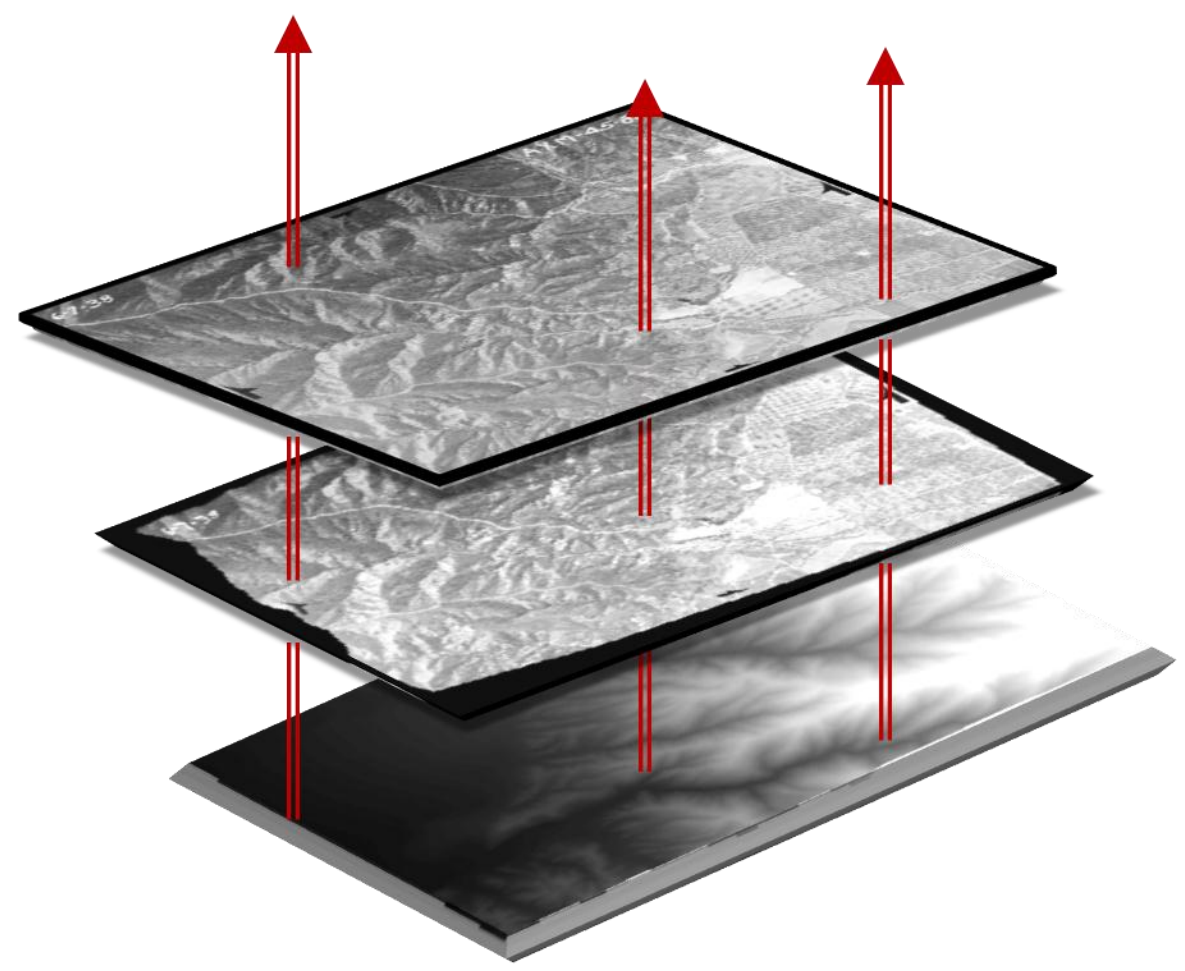

Figure 5-2 Data layers used to manually collect GCPs

The coordinates were organized in an $\mathrm{X}, \mathrm{Y}, \mathrm{Z}, \mathrm{COL}$, ROW format. The $\mathrm{X}$ and $\mathrm{Y}$ from the orthophoto were collected in UTM and the COL and ROW pairs from the unreferenced photos represent the pixel location which was calculated by multiplying measured coordinates in ArcGIS by the scanning resolution (in this case 1200 DPI.) The GCPs' format, as shown in Figure 5-3, had to be very specific because the Python-scripted tools read these values in a strict sequence. 


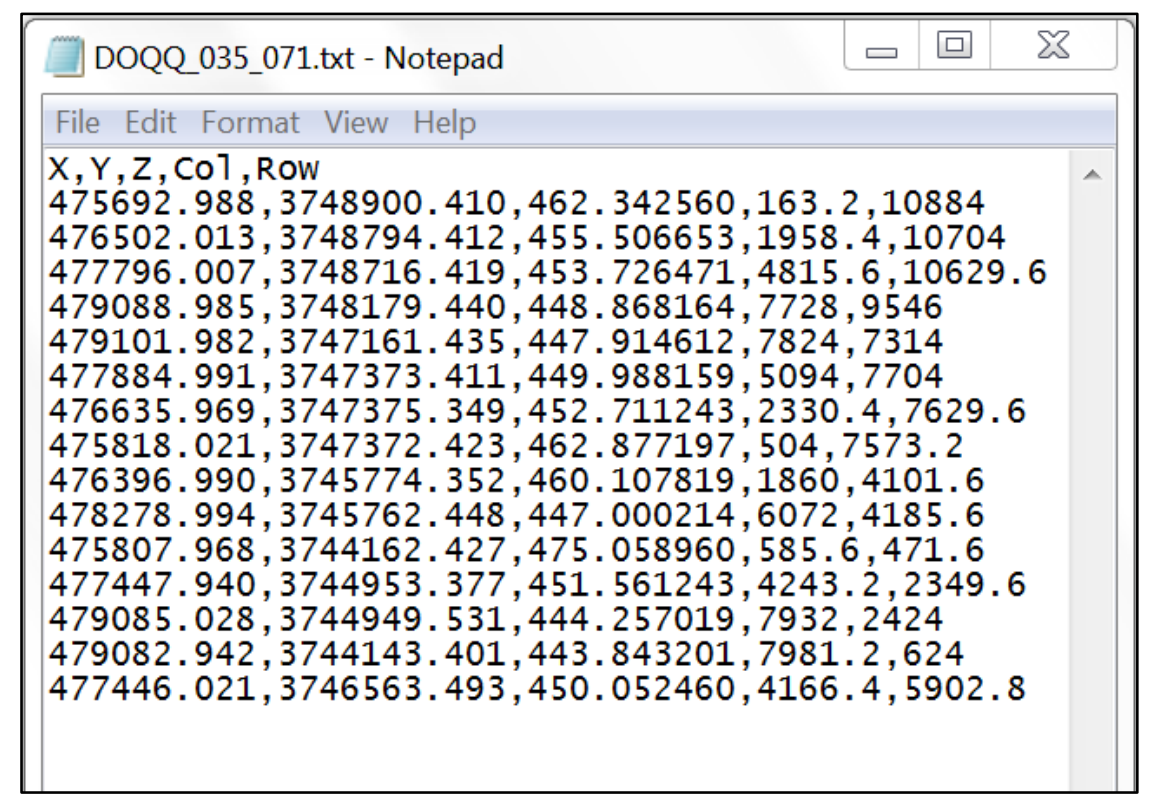

\section{Figure 5-3: Ground control point text file}

All of the GCPs were measured from reference imagery and DEM. They are considered ground truth since their errors were not modeled in calculating the RFM coefficients, which means their errors will propagate to the calculated RFMs and that the accuracy of the orthorectification relies on the accuracy of the reference data.

The accuracy of the orthorectified imagery relies on the quality and quantity of GCPs. For that reason, the collection of GCPs was a critical part of the project. The selected GCPs ideally should be evenly distributed throughout the image. Additionally, points should not be collected from features that could change, like shorelines, for example. Areas that have stationary features, like intersections or landmarks, are highly recommended (Verbyla and Chang, 1997).

Twenty to fifty GCPs were collected for each unreferenced photograph. The amount of GCPs to be collected is dependent on the RFM polynomial order to be used for the orthorectification. The 1 st order requires a minimum of six control points, the 2 nd order requires a minimum of fifteen control points and the 3rd order requires at least thirty 
control points. The GCPs should be collected mostly at intersections or landmarks that could be easily recognized on both images. Figure 5-4 shows an example of a point collected from a 1938 image and a DOQQ of the same area. It clearly demonstrates the challenges in the control point collection as ground surface had change dramatically.
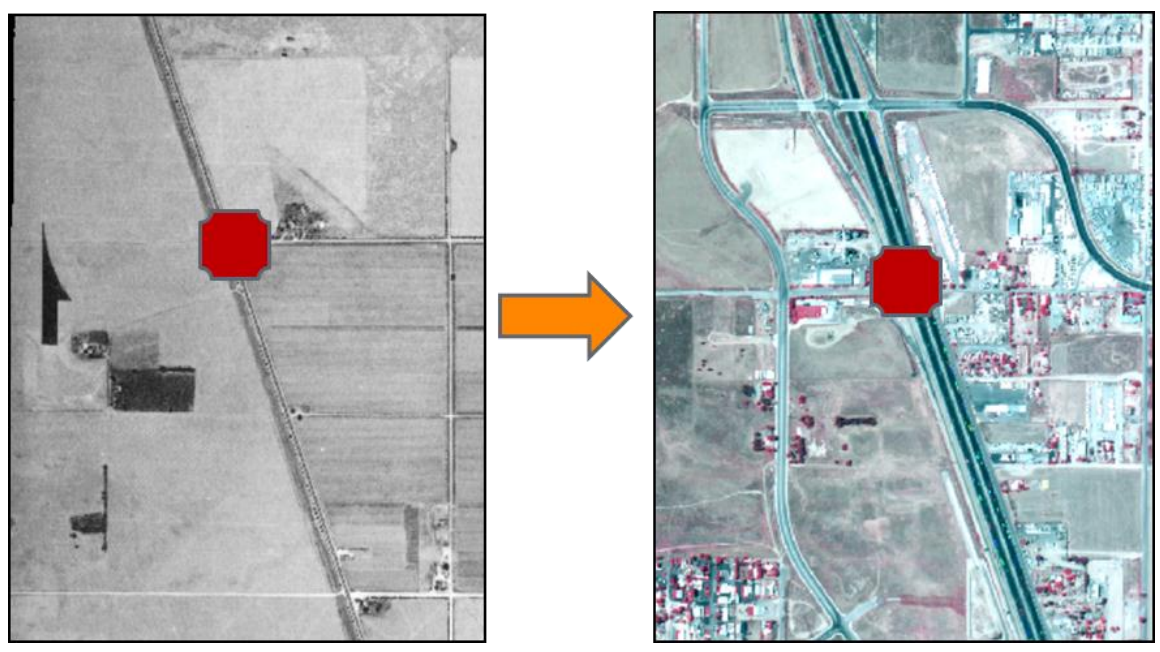

Figure 5-4: Collection of ground control points

\subsection{Rational Function Model Tools}

The RFM is a mathematical generalized sensor model. It uses a ratio of two polynomials to relate object point coordinates $(\mathrm{X}, \mathrm{Y}$, and $\mathrm{Z})$ to image pixel coordinates as physical sensor models do. There are two ways to calculate rational function coefficients (RFC): the terrain-independent approach and the terrain-dependent approach. The terrainindependent approach uses a rigorous camera model to generate virtual control points. It is commonly used in high resolution commercial satellite images. The terrain-dependent approach relies on manually collected control points. This project used the terrain dependent approach because a rigorous sensor model was not available. Such a model, however, could be developed with carefully selected GCPs to approximate the physical sensor model with high accuracy. This project's approach relied on terrain relief and the 
quality and the quantity of the GCPs. There should be a large number of GCPs welldistributed across an entire area of the image (Toutin, 2004).

Three transformation tools were used, one for each RFM order. Each tool was used to calculate a specific order of RFM from GCPs. The Python scripts read the ground control points' text file in the form of X, Y, Z, COL and ROW, and built a matrix from these values to calculate the coefficients. Figure 5-5 Shows an example of the RFM first order transformation tool.

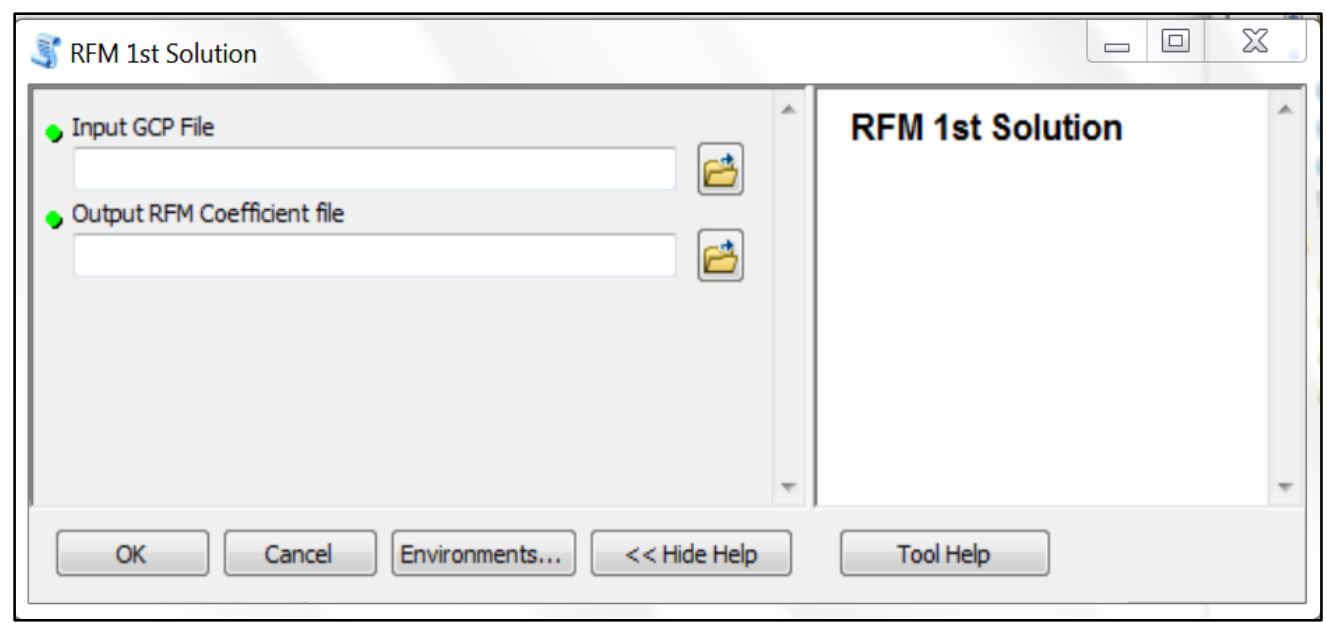

Figure 5-5: RFM transformation tool 


\subsection{Generating orthophotos using the tool}

After a RFM was resolved, the next step was to orthorectify the images using the orthorectification tool. Figure 5-6 shows the orthorectification tool used in the project. For this step, the Geospatial Data Abstraction Library (GDAL) version 18 was required. The orthorectification tool took the spatial reference from the DEM and the pixel values from the image to rebuild the image pixel by pixel using the RFM model previously calculated.

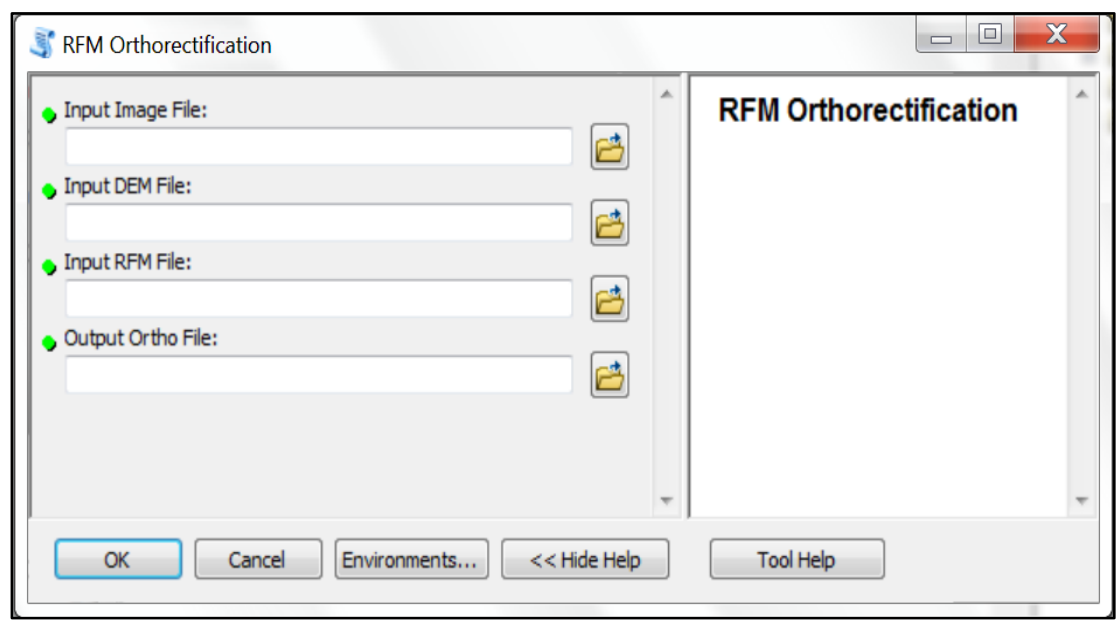

\section{Figure 5-6: Orthorectification tool}

Using the orthorectification tool some of the 1938 images that were collected over mountainous regions in Riverside County were orthorectified using different RFM orders to verify the quality of the orthorectification. This part of the project was to analyze differences on the different orthophotos in terms of RMSE values and distortions that could have been introduced by the RFM model. According to Okeke (2012) polynomial rectifications are not appropriate transformations for every type of image. The types of transformations vary according to the desired accuracy and the available number of GCPs; some high order polynomials can introduce distortions on the images. 


\subsection{Accuracy Assessment}

The accuracy of the orthorectification is one of the more important parts of the project. To assess the image accuracy, check points $(\mathrm{CP})$ were collected in the same manner as the GCPs. In Excel spreadsheets, the values of the CPs were normalized based on the RFM calculation. Given their ground coordinates, the row and column numbers of the check points were calculated using the RFMs derived from control points. The calculated row/column numbers were compared with measured row/column numbers and the root mean square error (RMSE) was calculated.

The RMSE compares the measured position on an image to the corresponding transformed position on the image. The difference between the measured positions and the transformed positions are known as the residual errors. The total error is calculated by taking the root mean square sum of all the residuals to compute the RMSE. It was used as a measure of the precision of the transformation. A perfect transformation would produce 0.00 (Smith, 2006), which most likely will never happen because there are always errors in measuring the GCPs but lower RMSE values are an indication of a good fitting between the transformation and the GCPs. A low RMSE value, however, doesn't necessarily mean that the model represents the physical imaging geometry accurately. The transformation may still contain significant errors resulting from a poorly selected GCP even with a small RMSE.

For this research project GCPs and CPs were manually collected for each image from Riverside County, CA because the camera geometry for the images was unknown and the RFM coefficients had to be calculated from GCPs. Since Harris County, Texas 
camera geometry was known, accurate GCPs and CPs were calculated using standard photogrammetry aerial triangulation method and were provided by the client.

\subsection{Summary}

This chapter focused on the main experiments of the project as well as the methodology conducted as part of the research. The purpose of the research was to conduct an accuracy assessment of the rational function model, to investigate the accuracy of the different RFM orders in different types of aerial images. For this, control points were collected from the unreferenced photos, the DOQQs, and DEM. The values were

organized in an X, Y, Z, COL, ROW format. Three transformation tools were used, one for each RFM order. The next step was to orthorectify the images using the orthorectification tool. Once an image was orthorectified, root square mean errors were calculated to perform an accuracy assessment of the RFMs. 



\section{Chapter 6 - Results and Analysis}

The accuracy assessment for the RFMs was the core component of the research project.

To assess the accuracy of the RFM model, check points (CP) were collected the same way the ground control points (GCP) were collected.

The first part of the project used images from Harris County, Texas to evaluate their accuracy assessment. Since these images' rigorous camera model was available, this part of the research project tested if the RFM could closely approximate the rigorous camera model. This part of the research required very accurate GCP. For this reason, the GCPs collected over Harris County, Texas were provided by one of the clients. The GCPs and CPs were generated from aerial triangulation with very high accuracy in comparison to the historical photographs from Riverside County. Table 6-1 shows the RMSE values of GCPs collected over Texas.

Table 6-1: RMSE values of GCPs collected over Texas

\begin{tabular}{|c|c|c|c|}
\hline Image & \multicolumn{3}{|c|}{ RMSE GCPs } \\
\hline & $\mathbf{1}^{\text {ST }}$ Order & $\mathbf{2}^{\text {nd }}$ Order & $\mathbf{3}^{\text {rd }}$ Order \\
\hline 1180223 & 0.108 & 0.048 & 0.042 \\
\hline 1180224 & 0.102 & 0.042 & 0.027 \\
\hline 552191 & 0.046 & 0.03 & 0.018 \\
\hline 552192 & 0.048 & 0.03 & 0.018 \\
\hline
\end{tabular}

Table 6-2: RMSE values of CPs collected over Texas

\begin{tabular}{|c|c|c|c|}
\hline Image & \multicolumn{3}{|c|}{ RMSE Checkpoints } \\
\hline & $\mathbf{1}^{\text {ST }}$ Order & $\mathbf{2}^{\text {nd }}$ Order & $\mathbf{3}^{\text {rd }}$ Order \\
\hline 1180223 & 0.105 & 0.078 & 0.066 \\
\hline 1180224 & 0.072 & 0.069 & 0.084 \\
\hline 552191 & 0.06 & 0.081 & 0.411 \\
\hline 552192 & 0.06 & 0.05 & 0.36 \\
\hline
\end{tabular}


The accuracy assessment of the images collected over Harris County, Texas used different orders of RFMs to approximate the rigorous sensor model. The RMSE values for the GCPs presented a very good fit between the GCPs and the transformation model for all of the tested RFM orders. Table 6-2 shows the RMSE values for the CPs, they showed a close approximation of the rigorous camera model by different orders of RFMs. All of the values obtained for the Texas images met the National Map Accuracy Standards.

The accuracy of the different RFM orders was also evaluated for two additional forest mountain areas in Riverside. To assess the accuracy of the RFM orders, CPs and GCPs were collected in the same manner. Fifty control points and fifteen checkpoints were collected for each photo. Table 6-3 and 6-4 show the resulting RMSE values for the GCPs and the CPs of the two images. After the RFM was solved using the GCPs collected with the Python-scripted tools of each RFM order for each photograph. The RMSE values of the check points of the aerial photographs were calculated using Excel to assess the accuracy of the derived RFM orders.

Table 6-3: RMSE values for the GCPs used to calculate different RFM orders

\begin{tabular}{|c|c|c|c|}
\hline Image & \multicolumn{3}{|c|}{ RMSE GCPs } \\
\hline & $\mathbf{1}^{\text {ST }}$ Order & $\mathbf{2}^{\text {nd }}$ Order & $\mathbf{3}^{\text {rd }}$ Order \\
\hline $045-083$ & 0.768 & 0.6 & 34.93 \\
\hline $045-084$ & 0.18 & 0.23 & 5.45 \\
\hline
\end{tabular}


Table 6-4: RMSE values for the CPs

\begin{tabular}{|c|c|c|c|}
\hline Image & \multicolumn{3}{|c|}{ RMSE Checkpoints } \\
\hline & $\mathbf{1}^{\text {ST }}$ Order & $\mathbf{2}^{\text {nd }}$ Order & $\mathbf{3}^{\text {rd }}$ Order \\
\hline $045-083$ & 1.096 & 1.10 & 17.43 \\
\hline $045-084$ & 5.124 & 0.716 & 17.1 \\
\hline
\end{tabular}

The results for the first order showed low RMSE values for both images (045-083 and 045-084); the GCPs and the CPs showed a very good fit between the RFM and the control points, and they displayed a satisfactory accuracy that met the National Map accuracy standards in the selection of the GCPs.

The second order showed low values on the RMSE of the GCPs and the CPs for both images; however, the orthorectified images displayed distortions on the images after their orthorectification. Figure 6-1 shows the image distortions of one of the orthorectified images that used the RFM second order. These distortions are associated with a phenomenon called asymptote surface, which is related to issues when the polynomial function in the denominator of the model equals to zero. When the denominator of an RFM approaches zero, the calculated image coordinates will approach infinity. That indicates that a good fit between GCPs and an RFM does not guarantee a good representation of the imaging geometry. 


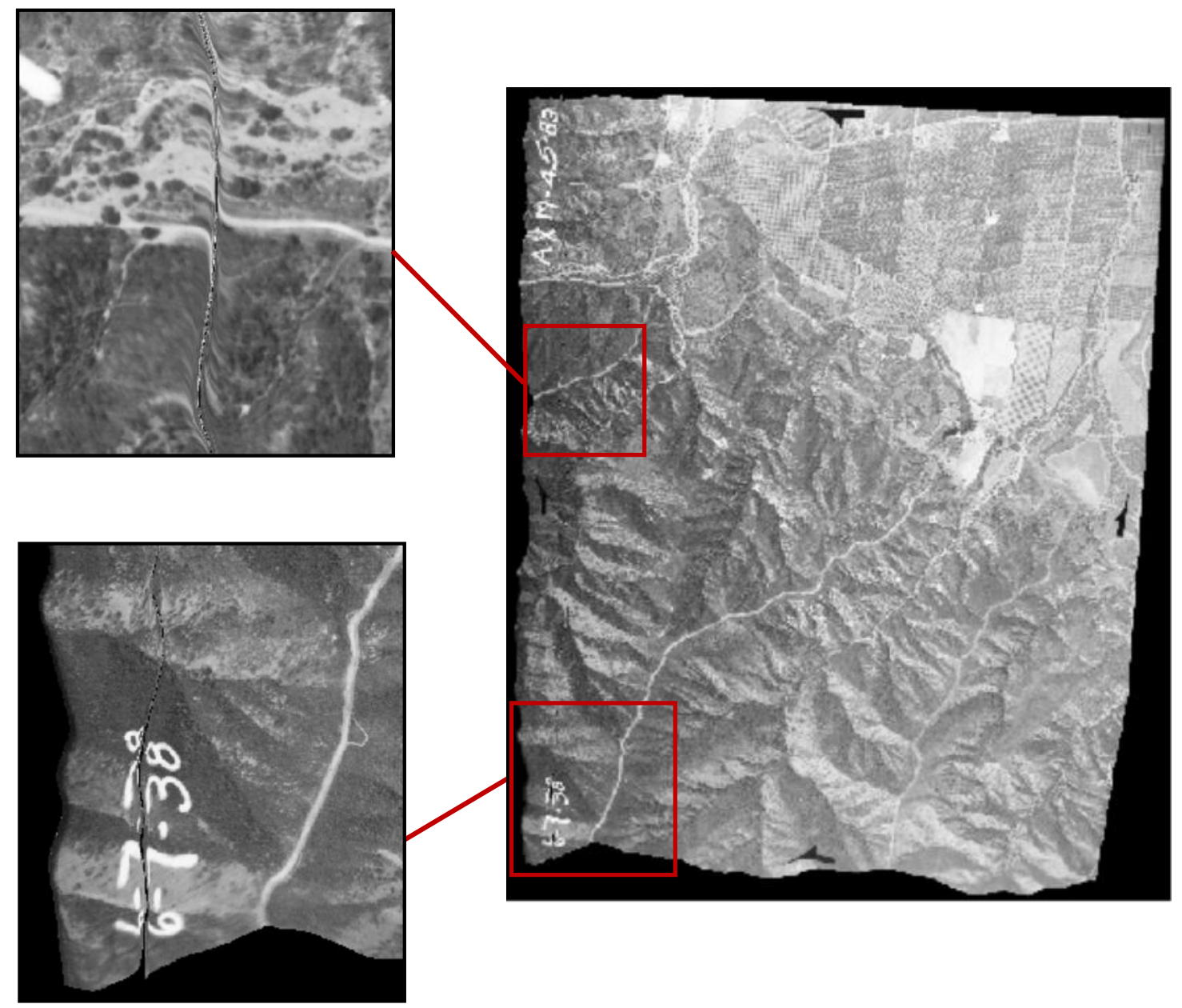

Figure 6-1: Asymptote distortion created by the RFM second order

The accuracy of the third order of the RFM not only presented very high RMSE values for GCPs and for CPs, but it also showed asymptote surface distortions on the orthorectified image, making it a less desirable RFM order to orthorectify images. Figure 6-2 shows distortions created by the third order RFM on one of the orthorectified images. The high RMSE indicated very poor fit between the control points and the RFM and a low accuracy of the GCPs. 


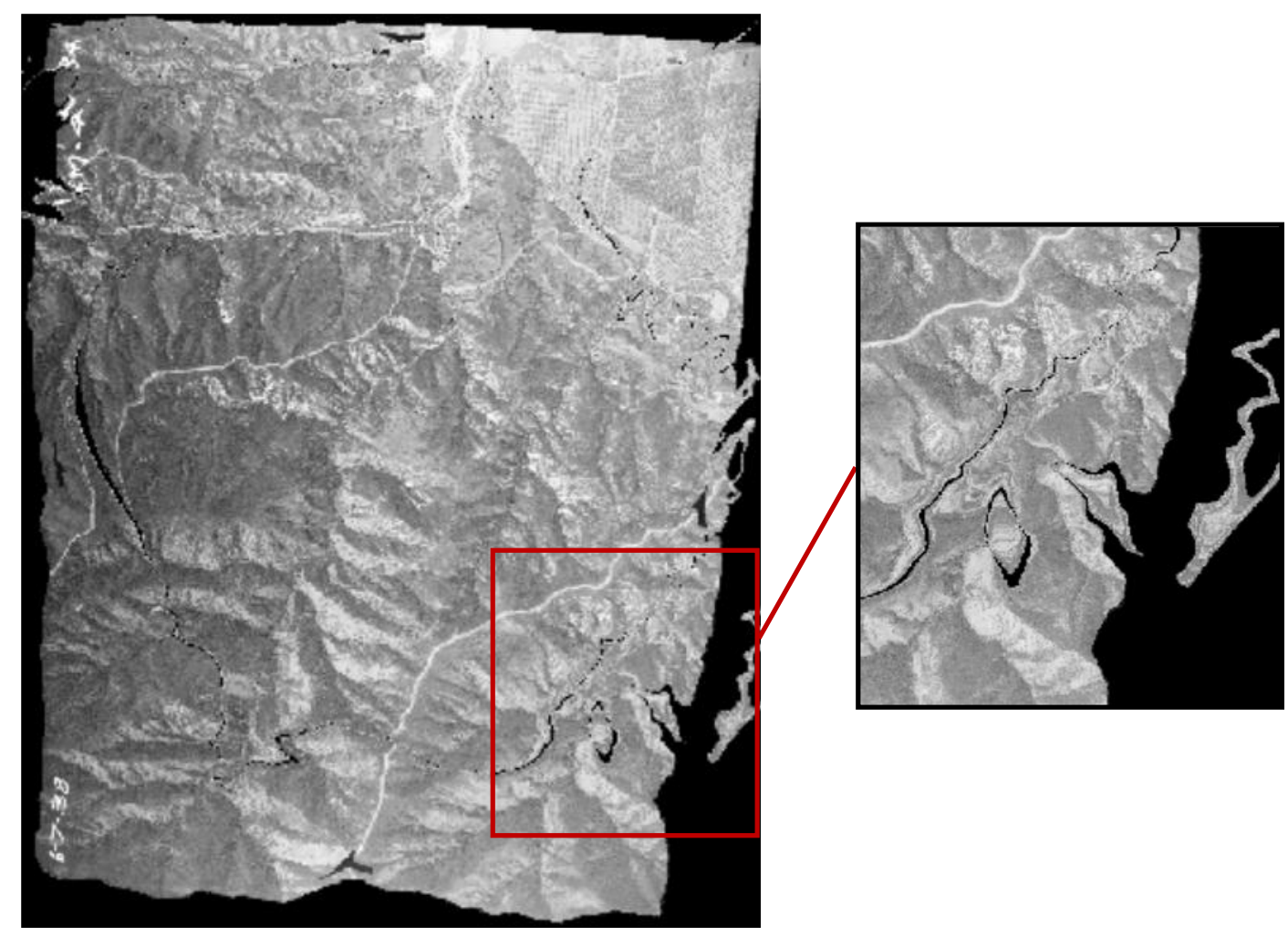

Figure 6-2: Asymptote distortion created by RFM third order

Figure 6-3 shows another comparison of the orthorectified image using the first order RFM and the DOQQ reference image. The same road was digitized using the DOQQ image and the RFM orthorectified image to compare the accuracy of the RFM orthorectification in mountain terrain. In general, the two lines match very well on the longest areas, but on close examination, there are positional discrepancies probably associated with noticeable ground surface change. This showed high accuracy on the mountain roads exhibited by the RFM orthorectified image. 


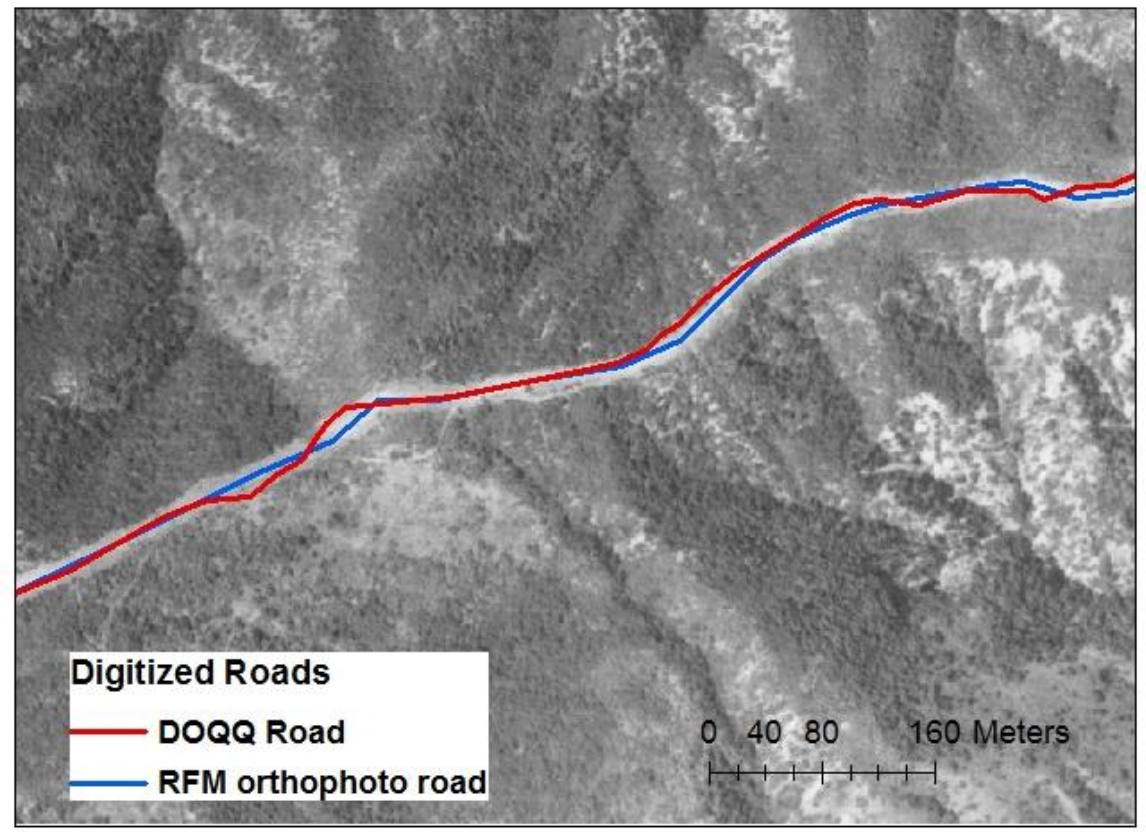

Figure 6-3: Digitized roads from the DOQQ and the RFM orthoimage

In this part of the results, the linear RFM calculation was solved using the GCPs

collected from each photograph with the Python-scripted tools, and the root mean square error (RMSE) values for six of the Riverside images were calculated. The RMSE values of the check points from each photograph were also calculated using Excel to assess the accuracy of the derived RFM. Table 6-5 lists the RMSEs of the CPs and the GCPs for the six photographs collected over Riverside County. Twenty control points and fifteen checkpoints were collected for each photo.

Table 6-5: GCPs and CPs RMSE of Riverside

\begin{tabular}{|c|c|c|}
\hline Photograph ID & RMSE from GCPs & RMSE from CPs \\
\hline $\mathbf{0 4 5 - 0 6 1}$ & 1.37 & 2.42 \\
\hline $\mathbf{0 4 5 - 0 6 3}$ & 2.58 & 5.71 \\
\hline $\mathbf{0 4 5 - 0 6 4}$ & 1.32 & 4.06 \\
\hline $\mathbf{0 3 5 - 0 7 1}$ & 2.34 & 5.06 \\
\hline $\mathbf{0 3 5 - 0 7 3}$ & 2.98 & 6.53 \\
\hline $\mathbf{0 3 5 - 0 7 5}$ & 3.24 & 5.92 \\
\hline
\end{tabular}


This imagery was collected over the City of Corona sixty years ago. The areas have forested mountains, agriculture fields, and urban developments. Urban development has changed the ground features in this area, and the changes in terrain made the collection of control points very difficult.

Although the project didn't require it, a comparison of the results with the National Map Accuracy Standards was created. The National Map Accuracy Standards state that 90 percent of all points tested must be within one-fiftieth of an inch on the map which would measure 33.33 feet - or 10.16 meters - on the ground at a scale of 1:20,000. Figure 6-4 shows that the overall RMSE results on the CPs of these six photographs showed that all of the images had errors lower than 10.16 meters, and that the accuracy of the orthorectified historical photographs met the mapping standards. Those results indicated an acceptable accuracy regarding the collection of the GCPs. The RMSE values for the GCPs indicated a good fit between the RFM and the GCPs.

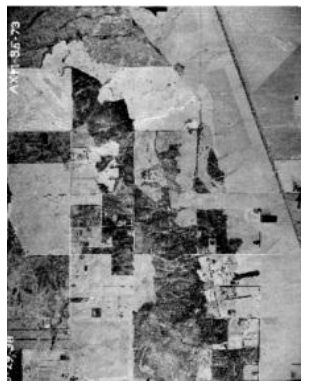

GCP 2.98

CP 6.53

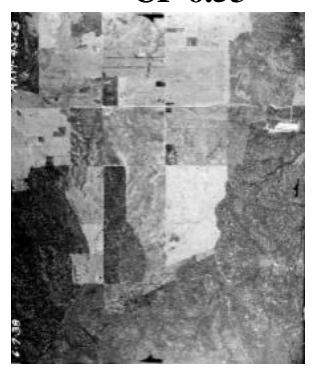

GCP 2.58

CP 5.71

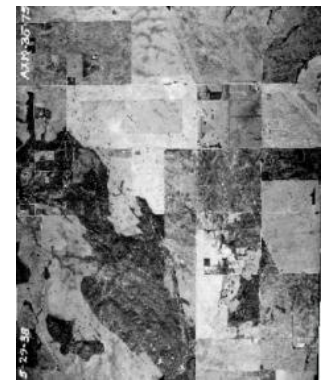

GCP 3.24

CP 5.92

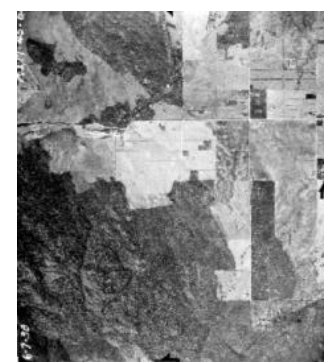

GCP 1.32

CP 4.06

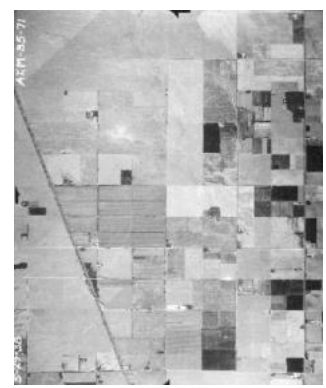

GCP 2.34

CP 5.06

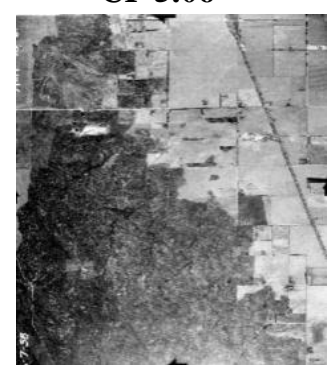

GCP 1.37

CP 2.42

Figure 6-4: Riverside images with their respective RMSE values 
The accuracy was also evaluated for the checkpoints of two of the Riverside images. Tables 6-6 show the RMSE overall calculations using the checkpoints for the RFM orthorectified image and the DOQQ. The coordinates were measured in UTM with a distance unit in meters.

Table 6-6: RMSE of the RFM orthophoto and the DOQQ for images 035-073 and 035-075

\begin{tabular}{|c|c|c|}
\hline Image & RMSE (m) & $\%<10.16$ \\
\hline $035-073$ & 2.02 & $100 \%$ \\
\hline $035-075$ & 1.63 & $100 \%$ \\
\hline
\end{tabular}

In these comparisons CPs collected on the first order RFM transformation were compared with CPs collected in the same areas on the DOQQ reference image. The RMSE values between the two were compared. This method produced low overall RMSE values in both images and met the Map Accuracy Standards. Both images 035-073 and 035-075 were more accurate than 10.16 meters (see appendix). This means 100 percent were within one-fiftieth of a map inch, which met the map accuracy standards; however, some of the points exhibit higher RMSE values than the Texas points which indicated problems with the collection of control points on the images. The topography of the area and the low image quality of the aerial data were probably responsible for these problems. 


\section{Chapter 7 - Conclusions and Future Work}

This research project tested the accuracy of the rational function model (RFM) to orthorectified different types of images - historical aerial images collected over mountain terrain with unknown camera geometry, and imagery collected over Texas with a rigorous camera model. The purpose of the project was to create an accuracy assessment of the quality of the ground control points, to orthorectify historical aerial photographs, and verify how closely the rational function model could approximate the rigorous camera model given highly accurate ground control points.

The accuracy assessment showed that the quality of the ground control points determined the accuracy of the model. Points collected manually using unreferenced imagery, DOQQs, and DEM - as with the Riverside County historical aerial imagery produced lower accuracy. Given accurate GCPs - as with the imagery over Texas - the three orders of the RFM can closely approximate the rigorous camera model.

In the case of the historical data in mountain terrain, the linear RFM obtained robust

and stable results that met the Map Accuracy Standards. However, the $2^{\text {nd }}$ and $3^{\text {rd }}$ order of the RFM suffered from asymptote surface, which was related to the low quality of the manually-collected ground control points. The rough terrain areas where these control points were collected experienced changes in the landscape over the last 60 years, which made the collection of high quality ground control points almost impossible. This resulted in a decline in accuracy of the RFM orthorectification. 


\subsection{Future Work}

Although the project fulfilled all the requirements, some areas could be improved in the future. A tool in ArcGIS that could collect ground control points when the camera geometry is unknown would be very helpful. Right now, ArcGIS 10 has an interface to orthorectify imagery, but it works only for imagery with available camera values.

The most critical part of the project was the collection of ground control points. The way the control points were collected was difficult, inconvenient, and added more chances to introduce human errors in the collection than a well-designed and simple interface. ERDAS Imagine has a user-friendly interface for the collection of ground control points. A future designer could help construct a new user-interface in ArcGIS to mimic ERDAS interfaces, so that a user has the different datasets side by side; can click a point that records the points as $\mathrm{x}, \mathrm{y}$ from the unreferenced image and $\mathrm{X}$, $\mathrm{Y}$ from the DOQQ on a table; and allows the user to overlay the DEM on top of the DOQQ for the collection of the $\mathrm{Z}$ values. Once the user collects all the control points, the interface would have a button to calculate the RMSE values per point, in order to tell the user the values that have low accuracy. This way, the user can recollect points easily and immediately. 


\section{Works Cited}

Buchwald, A. (2011). A Production Method for Conversion of Scanned Historic Aerial Imagery into Orthophotos Using the Rational Function Model (Unpublished master's thesis). University of Redlands, Redlands, California.

Esri. (2011). Fundamentals for Georeferencing a Raster Dataset. from ArcGIS Resource Center, Desktop 10: http://help.arcgis.com/en/arcgisdesktop/10.0/help/index.html\#//009t000000mn000 000

Fox, A. \& Czifersky, A. (2008). Unlocking the Time-Capsule of Historic Aerial Photography to Measure Changes in Antarctic Peninsula Glaciers. Photogrammetric Record, 23:51-68.

Hu, Y. Tao, C. \& Croitoru, A. (2004). Understanding the Rational Function Model: Methods and Applications. International Archives of Photogrammetry and Remote Sensing.

Kaichang, D., Ma, R. \& Li, R. (2001). Deriving 3-D from high resolution Ikonos Satellite images with rational functions. ASPRS Conference, St. Louis, Mo, April 25-27, 2001.

Ma, R. \& Buchwald, A. (2012). Orthorectifying Historical Aerial Photographs Using DLT. ASPRS 2012 Annual Conference Sacramento, California.

Morgan, J. L; Gergel, S. E. \& Coops, N. C. (2010). Aerial Photography: A Rapidly Evolving Tool for Ecological Management. BioScience 60, 47-59.

NGDA. (n.d.). National Geospatial Digital Archive, http://www.ngda.or/home.html, visited 10/8/2011. 
Smith, R. (2006). Choosing Control Points, RMS Error and Image Rectification. Retrieved from MicroImages: http://gisteam.de/ftp/Tutorial/rpcortho.pdf

Smith, R. (2011). Orthorectification Using Rational Polynomials. Retrieved from Orthorectification Tutorial: http://www.microimages.com/documentation/Tutorials/rpcortho.pdf

Sumerall, R. M \& Lloyd, T.F. (1994). GIS as a Design Tool for Biological Studies. Eighth Biennial Southern Silvicultural Research Conference, (pp. 36-41). Auburn, Al.

Tao, C. (2001). A Comprehensive Study of the Rational Function Model for Photogrammetric Processing. Photogrammetric Engineering \& Remote Sensing, $1347-1357$.

Tonolo, G. F. \& Poli, D. (2003). Georeferencing of Eros-A1 High Resolution Images with Rigorous and Rational Function Model. ISPRS workshop High Resolution Mapping from Space. Hanover, Germany.

Toutin, T. (2004). Review article: Geometric processing of remote sensing images: models, algorithms and methods. International Journal of Remote Sensing., Vol. 25(No. 10), 1893-1924.

Verbyla, D. L. \& Chang, K. (1997). Chapter 6: Image Rectification. Image Processing: A GIS Perspective. OnWord Press 1997. 


\section{Appendix A. Python Script for RFM Bilinear orthorectification tool}

\#import related modules

import numpy

import arcpy

import sys

import math

imgFName=sys.argv[1] \#Input image file name

demFName=sys.argv[2]

\#Input DEM file name

rfmFName=sys.argv[3]

\#Input transformation coefficient file name

orthoFName=sys.argv[4]

\#Output orthophoto file name

$\operatorname{minZ}=$ float $($ sys.argv[5])

demMeta=arcpy.Describe(demFName)

lower_left=demMeta.Extent.lowerLeft \#Lower left corner $\mathrm{x}, \mathrm{y}$. To find the exact value, use lower_left.X, lower_left.Y

upper_left=demMeta.Extent.upperLeft

\#Find out the DEM meta data about the image file

\#Assume all bands having the same spatial resolution, data type, and row/column

numbers

layerMeta=arcpy.Describe(demFName) \#Single layer data need not to be accessed by specifying the layer number.

demRows=layerMeta.height \#know how many rows and columns

demCols=layerMeta.width

$\mathrm{xReso}=$ layerMeta.meanCellWidth \#Spatial resolution along $\mathrm{x}$

yReso=layerMeta.meanCellHeight \#Spatial resolution along $\mathrm{y}$

\#Find out the raw aerial photograph information

layerMeta=arcpy.Describe(imgFName)

imgRows=layerMeta.height

imgCols=layerMeta.width

dataType=layerMeta.pixelType \#Data type such as unsigned 8-bit, signed integer 32-bit, and float 32

\#dataType=str(layerMeta.pixelType) \#Data type such as unsigned 8-bit, signed integer 32-bit, and float32

\#arcpy.AddMessage(dataType)

if dataType==u'U8': $\quad$ \#you could have problems with the script if your format is

different

arrayType=numpy.uint8

elif dataType $==u^{\prime} U 16$ :

arrayType $=$ numpy.uint 16

else: 
arrayType=numpy.uint 32

\#Read the image and DEM file data into the matrices so you can work on the image pixel by pixel

\#arcpy has problem when it reads large files. It seems there is a size limit of 100M. demMat=numpy.matrix (arcpy.RasterToNumPyArray(demFName), dtype=numpy.float32) \#Read into float number for calculation type of DEM float 32 imgMat=numpy.matrix (arcpy.RasterToNumPyArray(imgFName), dtype=arrayType $)$

\#Read the text file for transformation coefficients

rfmFile $=$ open $($ rfmFName, mode $=$ 'r')

rfmStr=rfmFile.readline()

if $\operatorname{rfmStr}<>$ RFM_Order=1 1 n':

sys.exit()

rfmStr=rfmFile.readline ()

xoffset=float(rfmStr.split('=')[1]) \#The $\mathrm{x}$ image coordinate of the image center

rfmStr=rfmFile.readline()

yoffset=float(rfmStr.split('=')[1])

rfmStr=rfmFile.readline()

\#The y image coordinate of the image

center

zoffset=float(rfmStr.split('=')[1])

rfmStr=rfmFile.readline()

coloffset=float(rfmStr.split('=')[1])

rfmStr=rfmFile.readline()

rowoffset=float(rfmStr.split('=')[1])

$\mathrm{rfmStr}=\mathrm{rfmFile}$.readline()

xScale=float $($ rfmStr.split('=')[1])

rfmStr=rfmFile.readline ()

yScale=float $($ rfmStr.split('=')[1])

rfmStr=rfmFile.readline()

zScale $=$ float $\left(\operatorname{rfmStr} \cdot \operatorname{split}\left({ }^{\prime}='\right)[1]\right)$

rfmStr=rfmFile.readline()

colScale $=$ float $\left(\operatorname{rfmStr} . \operatorname{split}\left({ }^{\prime}='\right)[1]\right)$

rfmStr=rfmFile.readline()

rowScale=float $\left(\mathrm{rfmStr} . \operatorname{split}\left({ }^{\prime}='\right)[1]\right) \quad$ \#The scale used to transform image coordinate to row/column number, split parse the data if it equal tell to separate the data

\#Read the coefficients

rfmMat=numpy.zeros(11,dtype=numpy.float $)$ \#Tweleve coefficients

for $\mathrm{i}$ in range $(0,11,1)$ : \#this is base on the numbers of the rfc text file

$\mathrm{rfmStr}=\mathrm{rfmFile}$.readline()

rfmMat[i]=float(rfmStr.split('=')[1])

rfmFile.close () \#Close the file

\#Prepare the output orthophoto matrix 
orthoMat=numpy.zeros((demRows,demCols $)$,dtype=arrayType $) \quad$ \#Assuming single layer

\#Loop through the DEM to produce the orthophoto

iRow $=0$

$\mathrm{iCol}=0$

for iRow in range $(0$, demRows, 1$)$ : \#teling it to start reading numbers starting with one in rows

for $\mathrm{iCol}$ in range $(0$, demCols, 1$)$ :

zGround $1=$ demMat[iRow,iCol]

if (zGround $1<\operatorname{minZ})$ :

continue \#continue mean skip the next stuff below and go back up to the if else:

xGround=((upper_left.X+iCol*xReso)-xoffset)/float(xScale)\#upper left means a point geometry on this script

yGround $=(($ upper_left.Y-iRow*yReso $)$-yoffset $) /$ float(yScale $)$

zGround $=($ zGround $1-$ zoffset $) / z$ Scale

xImage $=(\operatorname{rfmMat}[0]+\mathrm{rfmMat}[1] * \mathrm{xGround}+\mathrm{rfmMat}[2] * \mathrm{yGround}+\mathrm{rfmMat}[3] * \mathrm{zGround}) / \mathrm{fl}$ oat $(1.0+$ rfmMat $[8] * x$ Ground+rfmMat[9]*yGround+rfmMat[10]*zGround)

\#transformation equation

yImage $=(\operatorname{rfmMat}[4]+\mathrm{rfmMat}[5] * \mathrm{xGround}+\mathrm{rfmMat}[6] * \mathrm{yGround}+\mathrm{rfmMat}[7] * \mathrm{zGround}) / \mathrm{fl}$

oat $(1.0+$ rfmMat [8]*xGround+rfmMat[9]*yGround+rfmMat[10]*zGround)

collmage $=x$ Image $*$ colScale + coloffset $\quad$ \#Column number in decimal

rowImage $=$ imgRows-(yImage*rowScale+rowoffset) \#Row number in decimal \#imgRow=round(rowImage) \#Row number in closest integer, we

calculate the nearest neighbor point with the round value, we need to change this to use bilinear

\#imgCol=round(collmage) \#Column number in closest integer

if (collmage $<0$ or collmage $>$ (imgCols- 1$)$ or rowImage $<0$ or

rowImage $>$ (imgRows-1)):

orthoMat[iRow, iCol] $=0$

continue

else:

uLcol=int (math.floor(collmage $))$

uLrow=int(math.floor(rowImage))

uRcol=int(math.ceil(collmage))

$\mathrm{uRrow}=\mathrm{uLrow}$

1Lcol=uLcol

lLrow=int(math.ceil(rowImage))

$1 \mathrm{Rcol}=\mathrm{uRcol}$

lRrow $=1$ Lrow

\#if $\mathrm{uLrow}==0$ or $\mathrm{uLcol}==0$ : $\quad$ \#to tell the script to grab values from the DEM starting at 0 


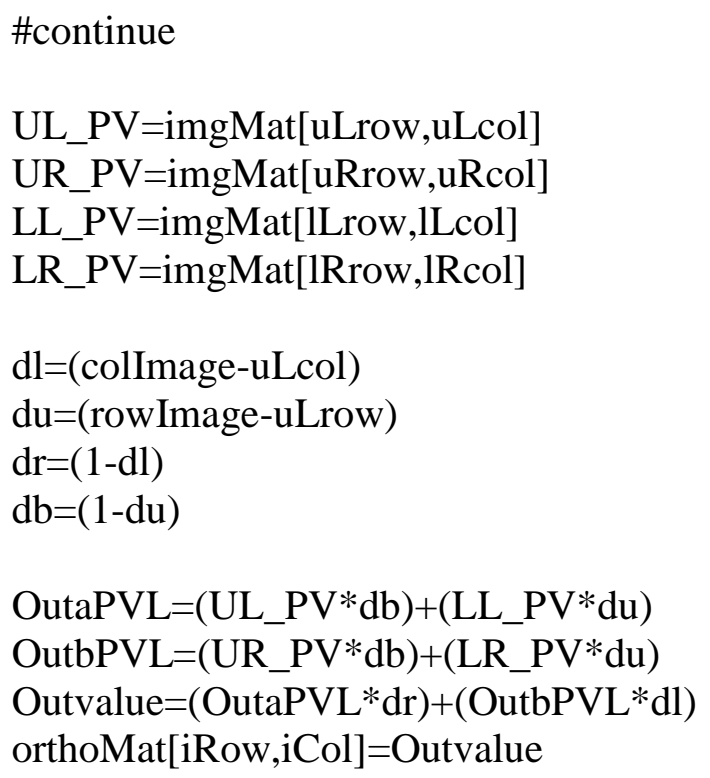

\#Convert to raster data with layer information

orthoOut=arcpy.NumPyArrayToRaster(numpy.array(orthoMat),lower_left,xReso,yReso) arcpy.env.overwriteOutput $=$ True \#Overwrite the output file. The default value is false .

\#The following two lines cannot be switched. The 'define projection management' is used for available data file so the raster has to be saved first orthoOut.save(orthoFName) arcpy.DefineProjection_management(orthoOut,demMeta.spatialReference)

arcpy.AddMessage(" ")

arcpy.AddMessage("Done!")

arcpy.AddMessage(" ") 


\section{Appendix B. Accuracy Spreadsheets}

\section{RMSE of the RFM orthophoto and the DOQQ for image 035-073}

\begin{tabular}{|c|c|c|c|c|c|c|c|}
\hline \multicolumn{2}{|c|}{$\begin{array}{l}\text { Orthorectified image } 035-073 \\
\text { RFM othophoto }\end{array}$} & \multicolumn{2}{|c|}{ DOQQ reference image } & \multicolumn{2}{|c|}{ Residual } & \multirow{2}{*}{$\begin{array}{c}\text { RMSE } \\
\text { Total RMSE: } 5.07\end{array}$} & \multirow{2}{*}{$\begin{array}{c}\text { Conversion } \\
\text { meters }\end{array}$} \\
\hline$x$ & $Y$ & $x$ & $\mathrm{Y}$ & $x$ & $Y$ & & \\
\hline 472815.84 & 48812.997 & 472813.518 & 3748814.092 & 2.321597 & -1.094765 & 2.566772884 & 1.026709153 \\
\hline 473631.386 & 3748767.522 & 473634.786 & 3748766.467 & -3.39983 & 1.054571 & 3.559624984 & 1.423849993 \\
\hline 475474.846 & 3748304.03 & 475473.644 & 3748314.69 & 1.202384 & -10.659929 & 10.72752598 & 4.29101039 \\
\hline 476306.918 & 3748409.97 & 476310.102 & 3748410.023 & -3.18397 & -0.052955 & 3.184415336 & 1.273766134 \\
\hline 473404.385 & 3747329.513 & 473405.598 & 3747330.644 & -1.21281 & -1.131465 & 1.658649666 & 0.663459867 \\
\hline 476106.344 & 3746560.525 & 476108.645 & 3746555.207 & -2.30072 & 5.31755 & 5.793933407 & 2.317573363 \\
\hline 474203.387 & 3746539.483 & 474203.073 & 3746539.259 & \begin{tabular}{|l|} 
\\
0.313661 \\
\end{tabular} & 0.2239 & 0.385375704 & 0.154150281 \\
\hline 475106.372 & 3747331.531 & 475103.226 & 3747335.729 & 3.145836 & -4.197928 & 5.245844415 & 2.098337766 \\
\hline 472614.385 & 3745740.556 & 472616.138 & 3745734.997 & -1.7531 & 5.558975 & 5.828856635 & 2.331542654 \\
\hline 473391.381 & 3744958.496 & 473396.66 & 3744954.475 & -5.27908 & 4.0206 & 6.635805947 & 2.654322379 \\
\hline 475115.393 & 3745753.486 & 475116.456 & 3745748.226 & -1.063 & 5.259629 & 5.365973592 & 2.146389437 \\
\hline 475806.904 & 3744960.965 & 475804.374 & 3744954.475 & 2.530415 & 6.490119 & 6.965963301 & 2.78638532 \\
\hline 475775.382 & 3744236.534 & 475776.338 & 3744238.039 & -0.95579 & -1.504972 & 2827342 & 0.713130937 \\
\hline 474110.427 & 3744145.554 & 474111.037 & 3744160.723 & -0.60987 & -15.168921 & 15.18117591 & 6.072470365 \\
\hline \multirow[t]{2}{*}{473746.423} & 3748030.523 & 473747.199 & 3748029.731 & \begin{tabular}{|c|}
-0.776 \\
\end{tabular} & 0.792391 & 1.109077675 & 0.44363107 \\
\hline & & & & & & 5.066121518 & 2.026448607 \\
\hline
\end{tabular}

\section{RMSE of the RFM orthophoto and the DOQQ for image 035-075}

\begin{tabular}{|r|r|r|r|r|r|r|r|}
\hline \multicolumn{2}{|l}{$\begin{array}{l}\text { Orthorectified image 035-075 } \\
\text { RFM othophoto (meters) }\end{array}$} & $\begin{array}{c}\text { DOQQ reference image } \\
\text { (meters) }\end{array}$ & \multicolumn{2}{c|}{$\begin{array}{c}\text { Residual } \\
\text { (meters) }\end{array}$} & RMSE & $\begin{array}{c}\text { Conversion } \\
\text { meters }\end{array}$ \\
\hline 469655.143 & 3748894.11 & 469652.379 & 3748892.4 & 2.764 & 1.665 & 3.226750843 & 1.290700337 \\
\hline 471446.679 & 3748921.708 & 471441.473 & 3748909.3 & 5.206 & 12.385 & 13.43468128 & 5.373872511 \\
\hline 472946.143 & 3749096.195 & 472953.49 & 3749091.2 & -7.347 & 4.946 & 8.856710733 & 3.542684293 \\
\hline 470152.648 & 3748105.694 & 470154.068 & 3748107.9 & -1.42 & -2.237 & 2.649635635 & 1.059854254 \\
\hline 472584.666 & 3748140.706 & 472590.878 & 3748137 & -6.212 & 3.679 & 7.219694246 & 2.887877698 \\
\hline 469719.627 & 3747076.761 & $469,713.53$ & 3747077.4 & 6.099 & -0.608 & 6.129230376 & 2.45169215 \\
\hline 471787.68 & 3746530.759 & $471,790.51$ & 3746531 & -2.831 & -0.244 & 2.841495557 & 1.136598223 \\
\hline 472610.661 & 3745742.728 & $472,611.85$ & 3745744.1 & -1.193 & -1.419 & 1.853863533 & 0.741545413 \\
\hline 470256.676 & 3745738.77 & $470,258.31$ & 3745738.3 & -1.63 & 0.46 & 1.693664666 & 0.677465866 \\
\hline 471404.696 & 3747421.752 & $471,405.97$ & 3747422.8 & -1.275 & -1.03 & 1.639062232 & 0.655624893 \\
\hline 470030.733 & 3744638.688 & $470,030.84$ & 3744640.1 & -0.105 & -1.408 & 1.411909699 & 0.56476388 \\
\hline 471167.672 & 3744611.829 & $471,170.27$ & 3744613.9 & -2.597 & -2.089 & 3.332916141 & 1.333166456 \\
\hline 473044.629 & 3744820.667 & $473,045.50$ & 3744821.9 & -0.87 & -1.265 & 1.535293132 & 0.614117253 \\
\hline 471505.713 & 3745724.732 & $471,506.48$ & 3745728.4 & -0.768 & -3.656 & 3.735794427 & 1.494317771 \\
\hline 473213.66 & 3746875.703 & $473,215.03$ & 3746874.7 & -1.371 & 1.005 & 1.699901762 & 0.679960705 \\
\hline & & & & & & 4.084040284 & 1.633616114 \\
\hline
\end{tabular}

